\title{
Precios de adjudicación y componentes del spread en la Bolsa de Valores de Lima
}

\author{
Luis Chávez-Bedoya, Carlos Loaiza Álamo \\ y Giannio Téllez De Vettori
}

RESUMEN

En este trabajo se analizan tres aspectos del mercado de acciones de la Bolsa de Valores de Lima (BVL): i) la relación de corto plazo entre la dinámica de precios, la dirección y el volumen del flujo de órdenes; ii) los componentes del spread y el punto de equilibrio del Libro de Órdenes Límite por acción, y iii) la dinámica de los precios, de la dirección de la orden y del volumen negociado por shocks de las mismas variables rezagadas. Los resultados econométricos para datos intradiarios del año 2012 muestran que la dinámica de corto plazo de las acciones más líquidas y menos líquidas del Índice General de la BVL se explica por la dirección del flujo de órdenes, cuyo impacto en el precio es temporal en ambos casos. 1chavezbedoya@esan.edu.pe

Carlos Loaiza Álamo es profesor de la Facultad de Administración y Finanzas en la Universidad Peruana de Ciencias Aplicadas (UPc), Lima, Perú. cloaizaa@pucp.pe

Giannio Téllez De Vettori es asistente de docencia en el Departamento de Economía de la Pontificia Universidad Católica del Perú e investigador afiliado en esan Graduate School of Business, Lima, Perú. giannio.tellez@pucp.pe 


\section{I}

\section{Introducción}

El mercado de valores peruano (MVP) presenta problemas en cuanto a su desarrollo sostenible como una alternativa de inversión, y a pesar de ello escasea la investigación básica y aplicada en la materia. No existe una cuantía significativa de investigaciones en que se trate teórica o empíricamente, o con ambos enfoques, el funcionamiento del MVP, y en este caso específico el mercado de acciones. Esta ausencia de investigación ha motivado el interés de este trabajo por contribuir a entender cómo funciona la dinámica de precios y la composición de los costos de ejecutar órdenes en el mecanismo de negociación que administra la Bolsa de Valores de Lima (BVL).

Los principales modelos de determinación del precio de los activos, es decir, el modelo de selección de carteras de Markowitz (1952) y el modelo de valoración de activos de Sharpe (1964) presentan al rendimiento esperado y al riesgo como determinantes de los precios. En ambos modelos los costos de ejecutar una transacción son iguales a cero, supuesto que es relajado en la teoría de la microestructura de los mercados financieros. Esta teoría se entiende como el estudio del proceso y de los resultados de la negociación de instrumentos financieros bajo reglas explícitas de intercambio o transacción (O’Hara, 1995).

El estudio de la prueba empírica de la teoría de la microestructura presenta dos grandes impedimentos: i) el acceso a microdatos de la negociación de acciones, y ii) la gestión de información voluminosa para las pruebas empíricas. Sin embargo, en esta oportunidad se ha podido acceder a dicha información con el fin de entender mejor cómo se comporta el mercado de acciones de la BVL. Dada esta situación favorable, se ha realizado una investigación del efecto de la dinámica de la adjudicación de precios y los componentes del margen de compraventa (bid-ask spread) del mercado de acciones de la BVL, tal como se ha analizado en los mercados accionarios más desarrollados del mundo. A nuestro entender, esta es la primera investigación en que se realiza una prueba empírica de la teoría de la microestructura para el mercado de renta variable peruano (MRVP) ${ }^{1}$.

El documento se organiza de la siguiente manera: en la sección II se describe el mecanismo de negociación en la BVL, mientras que en la sección III se brinda el marco teórico que presenta el estado del conocimiento; por su parte, la sección IV contiene la descripción y el tratamiento de los datos, así como los resultados de las estimaciones econométricas; por último, en la sección IV se exponen las conclusiones.

\footnotetext{
${ }^{1}$ La primera presentación teórica de microestructura para la Bolsa de Valores de Lima (BVL) se puede encontrar en Loaiza (2013).
} 


\section{II}

\section{Mecanismo de negociación de acciones en la BVL}

El estudio de la microestructura implica conocer las características del mecanismo de negociación en un mercado bursátil. Por esta razón, se describen aquí tales características respecto del mercado bursátil peruano.

La BVL presenta un mecanismo de negociación electrónico ${ }^{2}$ que agrega órdenes o propuestas ${ }^{3}$ de compra y venta en el Libro de Órdenes Límite (LOB por sus siglas en inglés). No hay especialistas o creadores de mercado ${ }^{4}$, por lo que la liquidez es provista únicamente por dicho libro. Existe una regla discriminatoria de precios; esta rige para todas las fases de negociación y consiste en la posibilidad de que una orden se ejecute por partes a precios diferentes. En este sentido, la prioridad de las propuestas (el orden en que serían ejecutadas) y sus respectivas adjudicaciones (calce entre oferta y demanda) se rigen por: i) la propuesta que "mejora el precio"5 en el LOB, y ii) la propuesta con un "mayor tiempo de exposición en el mercado"6.

La formación del precio inicial se da en una primera fase denominada preapertura, en la que el sistema de subastas recibe las propuestas sin posibilidad de ser canceladas. En esta fase se almacenan todas las expectativas del mercado sin que calcen. A partir de esta información, el sistema adjudica un precio en un

\footnotetext{
${ }^{2}$ El mecanismo de negociación se denomina ELEX, un software que permite la agregación de propuestas de compra y venta en el Libro de Órdenes Límite (LOB). Estas calzan automáticamente bajo reglas de mejor precio y tiempo en un proceso de subasta continua. Asimismo, las propuestas pueden ejecutarse a distintos precios.

${ }^{3}$ En este documento se utilizará indistintamente el término órdenes o propuestas.

4 Si bien hay una reglamentación del agente promotor que tiene funciones de creador de mercado, esta figura no se cumple en la realidad; véase Loaiza (2013).

${ }^{5}$ El que una propuesta mejore el precio en el LoB depende de si es compra o venta. Como la finalidad es que se reduzca el spread, una propuesta de compra mejora el precio si es mayor que la mayor propuesta de compra en el LOB. Inversamente, una propuesta de venta mejora el precio si es menor que la menor propuesta de venta en el LOB. ${ }^{6}$ Que una propuesta tenga un mayor tiempo de exposición en el mercado hace referencia a que se prioriza a quien inicia primero la propuesta; en este sentido, se le da prioridad al que origina una orden con mayor antelación.
}

"período de tiempo variable"7 a fin de que las propuestas pasen a ser ejecutadas.

La siguiente fase es la de negociación continua, en la que los operadores (traders) ingresan propuestas a la espera de que calcen automáticamente. Estas propuestas son órdenes límite con las que se puede comprar o vender especificando la cantidad, el precio y un período de exposición de la propuesta. En la orden límite no se pueden ingresar precios que superen el límite mínimo de variación (tick) de 0,01, un límite máximo de variación de un $15 \%$ para valores nacionales y del $30 \%$ para valores extranjeros, siendo estas variaciones referidas al último precio adjudicado del día anterior ${ }^{8}$. En el mecanismo de negociación de la BVL no se considera la posibilidad de órdenes de mercado, las que se caracterizan por no ejecutarse a un precio límite. Los operadores podrán visualizar en el libro un mercado por precio, tanto por parte de la compra (bid) como de la venta (ask). A partir de las órdenes límite recibidas se determina un mejor precio, que los operadores participantes en el LOB pueden visualizar de forma agregada (no individual). La diferencia entre los mejores precios bid y ask formará el spread.

La última fase es la de cierre, que funciona de manera similar a la preapertura determinando un precio de cierre de las acciones en un rango arbitrario de tiempo 9 . Una vez determinado dicho precio, toda propuesta de compra o de venta que se ingrese se transa a ese valor. Así, la formación del precio de cada acción responde a la demanda agregada neta (compras menos ventas) de cada acción que se presenta en cada momento de la negociación continua. En esta dinámica del precio pueden existir problemas de información asimétrica entre los inversionistas e inmediatez en la ejecución de sus transacciones; estos problemas generan costos que en la literatura de la microestructura se denominan "fricciones", debido a que repercuten en la formación de precios.

\footnotetext{
${ }^{7}$ Esto quiere decir que la primera adjudicación del día se encuentra en un intervalo de tiempo de +/- 2 minutos, tomando como referencia la hora de inicio de la siguiente etapa: la fase de negociación continua. ${ }^{8}$ Para todas las fases de negociación.

${ }^{9}$ Esto quiere decir que la última adjudicación en la fase de negociación se encuentra en un intervalo de tiempo de $+/-2$ minutos, tomando como referencia la hora de inicio de la siguiente etapa: la fase de cierre.
} 


\section{III}

\section{Modelos microestructurales}

En esta sección se revisan los modelos teóricos y empíricos basados en la teoría microestructural del mercado bursátil, y a partir de ellos se podrá determinar el mejor enfoque para explicar la negociación de acciones en la BVL.

\section{Algunos modelos basados en el enfoque de microestructura del mercado bursátil}

Con respecto al estado actual de conocimientos, el trabajo de Demsetz (1968) marca el inicio de la investigación en el campo de la microestructura, analizando la determinación de los precios en los mercados de acciones. Este autor incorpora el problema de inmediatez en la negociación, debido a la existencia de agentes con necesidades de liquidez (impacientes) y agentes sin necesidades monetarias (pacientes) que llegan al mercado en diferentes momentos de la negociación.

Garman (1976) es el primero que modela un proceso de negociación caracterizado por la presencia de un agente que gestiona un inventario de acciones y efectivo (cash). Su modelo se caracteriza por desequilibrios temporales de los flujos de órdenes de compra y venta (Demsetz, 1968), que producen incertidumbre con respecto al tiempo esperado de la llegada de una orden. Estos desequilibrios justifican la presencia de un creador de mercado que resuelva el problema de la incertidumbre en relación con el tiempo de llegada de una orden. El creador de mercado resuelve el problema ofreciendo acciones cuando la contraparte compra, y efectivo cuando la contraparte vende.

El modelo de Stoll (1978) también parte del problema de inmediatez y define al creador de mercado como un proveedor de servicios de liquidez o negociación. Este agente procurará compensar los costos de ofrecer inmediatez a través del spread pagando un menor precio a quienes desean comprar activos, y vendiendo a mayor precio a los que quieren vender activos. En este modelo, Stoll desagrega los costos en: i) costos de mantenimiento; ii) costos de procesamiento ${ }^{10}$, y iii) costos de información. Sin embargo, el autor se enfoca en el costo de mantenimiento, asumiendo que el mercado es competitivo y los demás costos son iguales a cero.

10 Stoll (1978 y 2000) considera como costos de procesamiento a aquellos relacionados con el ruteo o transmisión electrónica de órdenes, ejecución y liquidación de operaciones.
Glosten y Milgrom (1985) modelan el problema de información asimétrica que genera costos de selección adversa en la negociación de acciones. En el modelo se asume la llegada secuencial de operadores con información privada (insider traders) y operadores que requieren liquidez (liquidity traders) en un mercado de competencia perfecta. Los creadores de mercado revisan sus precios según la información que extraen del flujo de órdenes, utilizando un proceso de aprendizaje bayesiano. En este modelo se extrae información de la dirección de la orden y no de su tamaño. Un segundo trabajo relevante, en que se modela el problema de información asimétrica, es el de Kyle (1985), donde se asume el supuesto de equilibrio de expectativas racionales, subastas a precio único (no hay spread) y un mercado de competencia imperfecta. Así, el agente informado actúa estratégicamente anticipando las reacciones de los otros agentes. El resultado es que los costos de transacción de los agentes no informados son equivalentes a los beneficios de los agentes informados.

Stoll (2000) diferencia los costos de operar en dos clases: las denominadas "fricciones reales" y las "fricciones informativas". Ambas clases afectan a la formación de los precios. En el primer caso, el creador de mercado procura compensar los costos de procesamiento de órdenes y de inventario, así como obtener rentas de su poder de mercado, mientras que en el segundo es un intermediario que redistribuye la riqueza entre agentes informados y no informados, asumiendo solamente costos de selección adversa. Luego, Stoll (2000) atribuye los cambios transitorios en los precios a los problemas de fricción real, y los cambios permanentes en los precios, a problemas de fricción informativa.

Hasta aquí, la teoría de la microestructura se ha centrado en mecanismos en los que existe un creador de mercado. Sin embargo, la negociación de acciones no siempre es promovida por dicho agente, sino que también existe la posibilidad de que sea el LOB el proveedor de liquidez. El problema de los modelos del LOB es que solo se han desarrollado teóricamente (Rosu, 2009; Foucault, Kadan y Kandel, 2001); no obstante, Glosten (1994) desarrolla un modelo teórico del LOB con problemas de información asimétrica, donde el comportamiento del inversor se modela a partir de una ecuación de revisión de precios, la que puede ser estimable empíricamente y determinará el ingreso de órdenes límite. 
Jong, Nijman y Röell (1996) analizan el efecto en los precios durante un mismo día de negociación (intradiarios) y de los componentes del spread bid-ask de la Bolsa de París a partir del modelo de Glosten (1994). La prueba empírica consiste en un modelo econométrico donde el spread se descompone en dos factores, uno provocado por costos de procesamiento de órdenes y otro por información asimétrica.

Jong, Nijman y Röell (1996) desarrollan dos estimaciones: en una primera estimación, utilizando mínimos cuadrados ordinarios (MCO) para analizar el efecto inmediato en los precios, y en la segunda, empleando un modelo de autorregresión vectorial (VAR) para analizar si el efecto en el precio es permanente mediante la metodología propuesta por Hasbrouck (1991a). En este sentido, en Jong, Nijman y Röell (1996) se concluye que el costo de procesamiento es mayor, y los costos de selección adversa menores en operaciones más pequeñas. Además, se encuentra que el efecto de la dirección de las órdenes (compra o venta) en los precios en períodos posteriores es permanente.

A partir de esta revisión de la literatura, se puede concluir que en el mecanismo de negociación de acciones puede haber costos de operación que están compuestos por costos de procesamiento y de selección adversa. Luego, dadas las características descritas de la negociación en la BVL, se considera que el modelo que más se ajusta es el basado en el LOB. En relación con este tipo de modelos, una propuesta teórica que ha sido contrastada es el modelo de Glosten (1994). Este contraste empírico es desarrollado por Jong, Nijman y Röell (1996) siguiendo la metodología econométrica planteada por Hasbrouck (1991a). En la presente investigación se contrastará este modelo. A continuación se brinda una presentación del modelo de Glosten (1994) y extendido por Jong, Nijman y Röell (1996).

\section{Modelo de Glosten (1994) y extendido por Jong, Nijman y Röell (1996)}

Glosten (1994) desarrolla un modelo teórico que asume un LOB, precios discriminatorios y ausencia de creador de mercado. Tres características peculiares del modelo de negociación de la BVL. Como se ha descrito en la sección III.1, en este trabajo se modela el comportamiento del inversor y las características del equilibrio en un LOB cuando ingresa un gran número de órdenes límite. El inversor decide ingresar una orden de compra si la valoración marginal del precio es mayor o igual al precio del activo, y el equilibrio del LOB se caracteriza por un beneficio esperado de cero, donde - en un contexto de fuerte selección adversa— las pérdidas de unos agentes son los beneficios de los agentes informados. Luego, Jong, Nijman y Röell (1996) hacen una extensión al desarrollar una ecuación estimable del modelo teórico de Glosten (1994), incorporando costos de procesamiento. A continuación, se presenta la derivación de la ecuación de la dinámica de adjudicación del precio de las acciones, y los componentes del spread de precios.

En el modelo original de Glosten (1994) no existen costos de procesamiento de órdenes y se asumen transacciones iniciadas por el comprador. Sea $R(q)$ la ecuación de "revisión de precios", denotada por el precio ejecutado por un comprador con una orden de tamaño $q$, por sobre el valor esperado ex ante de su orden. En el modelo de Glosten (1994), este nivel $q$ representa el nivel mínimo para que se adjudique el precio ${ }^{11}$, siendo el valor marginal de una transacción de tamaño $q$ determinada por la siguiente regla:

$$
R^{\prime}(q)=E_{z}(g(Z) \mid Z \geq q)
$$

donde $g(Z)$ es la revisión de la mejor estimación pública del valor de la acción cuando se conoce que el comprador ejecutará una orden en el mercado de tamaño $Z$. $E_{z}$ denota la esperanza que se toma con respecto a la distribución del tamaño de la transacción $Z$. Se asume que esta distribución es exponencial, de modo que:

$$
F_{z}(z)=1-e^{\frac{-z}{\alpha}}
$$

La revisión de precios se describe como el cambio en las expectativas del verdadero valor de la acción, debido a una operación de tamaño Z. Para simplificar, se supone que esta relación es lineal:

$$
g(Z)=g_{0}+g_{1} Z
$$

Bajo estos supuestos, la relación de precio marginal es:

$$
R^{\prime}(q)=g_{0}+g_{1} E_{z}(Z \mid Z \geq q)=g_{0}+g_{1}(q+\alpha)
$$

donde la última igualdad se desprende de las propiedades de la distribución exponencial, siendo $\alpha$ el tamaño

\footnotetext{
${ }^{11}$ En la BVL, para que una operación marque precio, esta debe al menos ser mayor o igual que una Unidad Impositiva Tributaria (UIT), ya que de lo contrario, si bien la operación se realiza, ella no cambiará el precio.
} 
promedio de la transacción. Luego, Jong, Nijman y Röell (1996) proponen una extensión del modelo de Glosten (1994), introduciendo un costo de procesamiento de órdenes en el esquema de precio marginal. Sea la función de costo de procesamiento de órdenes, denotado por $C(q)$, la siguiente:

$$
R^{\prime}(q)=C^{\prime}(q)+g_{0}+g_{1}(q+\alpha)
$$

Integrando (5) y dividiendo por $q$ se obtiene el precio medio:

$$
\begin{gathered}
\int R^{\prime}(q) d q=\int C^{\prime}(q) d q+\int\left(g_{0}+g_{1} \alpha\right) d q+\int g_{1} q d q \\
R(q)=C(q)+\left(g_{0}+g_{1} \alpha\right) q+g_{1} \frac{q^{2}}{2} \\
\frac{R(q)}{q}=\frac{C(q)}{q}+\left(g_{0}+g_{1} \alpha\right)+\frac{1}{2} g_{1} q
\end{gathered}
$$

Para simplificar, se supone que el costo medio de procesamiento de pedidos es una función cuadrática de $q^{12}$, esto es $C(q)=c_{0} q+c_{1} q^{2}$. Por ende,

$$
\frac{R(q)}{q}=\frac{\left(c_{0} q+c_{1} q^{2}\right)}{q}+\left(g_{0}+g_{1} \alpha\right)+\frac{1}{2} g_{1} q
$$

Con lo que,

$$
\frac{R(q)}{q}=c_{0}+c_{1} q+\left(g_{0}+g_{1} \alpha\right)+\frac{1}{2} g_{1} q=R_{0}+R_{1} q(10)
$$

donde $R_{0}=c_{0}+g_{0}+g_{1} \alpha$ y $R_{1}=c_{1}(1 / 2) g_{1} . R_{0}$ captura los determinantes del precio promedio que no poseen relación con la cantidad adjudicada, en cambio $R_{1}$ captura los determinantes que poseen una relación directa con el monto operado. Finalmente, del modelo de Glosten (1994) se puede derivar la siguiente descomposición del spread bid-ask:

$$
C S A=\left(g_{0}+g_{1} \alpha\right)+\frac{1}{2} g_{1} q
$$

\footnotetext{
12 Cabe notar que esto implica una función cuadrática de costos con intercepto cero.
}

$$
C P O=c_{0}+c_{1} q
$$

donde CSA es el costo de selección adversa y CPO el costo de procesamiento de la orden. Para la aplicación empírica de este modelo, se introduce la siguiente notación de Jong, Nijman y Röell (1996):

$p_{t}=$ logaritmo del precio de adquisición (precio promedio pagado por acción).

$q_{t}=$ cantidad (número de acciones transadas).

$Q_{t}=$ dirección de la transacción ${ }^{13}$.

$y_{t}=$ valor esperado de la acción antes de la operación.

$\epsilon_{t}=$ cambio observado del valor de las acciones.

En el modelo empírico se establece que el precio de la transacción es igual al valor esperado de la acción antes de la operación, más la prima de precio promedio, $R(q) / q$, dada por la ecuación (10). Al igual que en Madhavan, Richardson y Roomans (1994), se añade un error en el precio al azar, $u_{t}$, que captura otras influencias en el precio de la transacción, como por ejemplo, la discrecionalidad en la adjudicación del precio.

Se considera que $u_{t}$ no está correlacionado con otras variables en la ecuación de precios. Además, se asume que la adjudicación del precio es dada por la dirección de la propuesta y el flujo de orden, propuestos en un período anterior a la ejecución $(t-1)$ revelando información al mercado. Por lo tanto, la ecuación de fijación de precios se convierte en:

$$
p_{t}=y_{t}+\left(R_{0}+R_{1} q_{t-1}\right) Q_{t-1}+u_{t}
$$

La revisión de precios puede ser modelada por el cambio en el valor esperado $y_{t}$, además de las operaciones, dirección y flujo de orden futuros, dado el precio adjudicado de la siguiente forma:

$$
y_{t+1}=y_{t}+\left(g_{0}+g_{1} q_{t+1}\right) Q_{t+1}+\in_{t+1}
$$

\footnotetext{
${ }^{13}$ Es importante analizar la dirección de la orden, debido a que esta propone información sobre la intención de obtener o deshacerse de acciones, impactando en la demanda (compra) u oferta (venta) del LoB. Si la operación es iniciada por una orden de compra, la dirección de la transacción será de signo positivo y tomará valor “+1". Si la operación es iniciada por una orden de venta, la dirección de la transacción será de signo negativo y tomará valor "-1". La dirección de la orden de compra se asume positiva debido a que una mayor demanda impulsaría un alza en el precio. Por el contrario, la dirección de la orden de venta se asume negativa, debido a que una mayor oferta impulsaría una disminución en el precio.
} 
La ecuación (14) se deriva de la ecuación (3), siendo $\epsilon_{t}$ la información pública que se encuentra entre las transacciones $t$ y $t+1$, pero no está relacionada con la transacción actual. Al operar con la ecuación (14) se tiene:

$$
\begin{gathered}
y_{t+1}-y_{t}=\left(g_{0}+g_{1} q_{t+1}\right) Q_{t+1}+\in_{t+1} \\
\Delta y_{t+1}=\left(g_{0}+g_{1} q_{t+1}\right) Q_{t+1}+\in_{t+1}
\end{gathered}
$$

Y si se itera un período hacia atrás, se tiene:

$$
\Delta y_{t}=\left(g_{0}+g_{1} q_{t}\right) Q_{t}+\in_{t}
$$

Ahora, presentada en términos de variaciones la ecuación (13):

$$
\Delta p_{t}=\Delta y_{t}+\left(R_{0}+R_{1} \Delta q_{t-1}\right) \Delta Q_{t-1}+\Delta u_{t}
$$

Se reemplaza (17) en (18):

$$
\begin{gathered}
\Delta p_{t}=\left(g_{0}+g_{1} q_{t}\right) Q_{t}+\in_{t}+\left(R_{0}+R_{1} \Delta q_{t-1}\right) \Delta Q_{t-1}+\Delta u_{t} \\
\Delta p_{t}=\left(R_{0}+R_{1} \Delta q_{t-1}\right) \Delta Q_{t-1}+\left(g_{0}+g_{1} q_{t}\right) Q_{t}+e_{t}
\end{gathered}
$$

donde $e_{t}=\epsilon_{t}+\Delta u_{t}$. La interpretación de la ecuación (20) se da de la siguiente manera: los coeficientes de las variables en "diferencia" son el intercepto y la pendiente del precio medio, mientras que los coeficientes de los niveles son estimados del intercepto y la pendiente de la función de revisión de precios. En otras palabras, el efecto en variaciones es el efecto por negociación (trading), y el efecto en niveles es el efecto por la adjudicación (liquidez).

La ecuación por estimar se obtiene reordenando (20):

$$
\begin{gathered}
\Delta p_{t}=c+R_{0} \Delta Q_{t-1}+R_{1} \Delta\left(q_{t-1} Q_{t-1}\right) \\
+g_{0} Q_{t}+g_{1} q_{t} Q_{t}+e_{t} \\
c_{0}=R_{0}-g_{0}-g_{1} \alpha \\
c_{1}=R_{1}-(1 / 2) g_{1}
\end{gathered}
$$

La ecuación (21) es la ecuación de la dinámica de precios de adjudicación; se incluye en esta ecuación la constante $c$ para capturar la rentabilidad media entre las transacciones (es decir, una media no nula de $e_{t}$ ). Las ecuaciones (22) ${ }^{14}$ y (23) son los determinantes de los costos del procesamiento de la orden.

${ }^{14} \alpha$ es el tamaño promedio de la transacción, dividido entre 2.

\section{IV}

\section{Prueba empírica}

\section{Datos Utilizados}

La estimación del modelo se realizará con los datos de la negociación electrónica de la BVL en el período 2012, con periodicidad intradiaria (operación por operación). Los activos elegidos para analizar son las cinco acciones más líquidas ${ }^{15}$ y las cinco menos líquidas ${ }^{16}$ de la

\footnotetext{
15 Volcan "B" (VOLCABC1), Rio Alto Minig (RIO), Ferreycorp (FERREYC1), Cerro Verde (CVERDEC1) y ADR Buenaventura (BVN).

16 Austral Group (Austrac1), El Brocal (BRocalc1), Agro. Ind. Pomalca (POMALCC1), Edelnor (EDELNOC1) y Scotiabank (SCOTIAC1).
}

cartera del Índice General de la Bolsa de Valores de Lima (IGBVL) ${ }^{17}$.

Las variables trabajadas fueron las siguientes:

i) Variación del precio $\left(\Delta p_{t}\right)$ : es el diferencial del logaritmo del precio de adquisición con respecto al precio anterior.

ii) Dirección de la transacción $\left(Q_{t}\right)$ : si la operación es iniciada mediante una orden de compra, esta será

\footnotetext{
17 El IGBVL está compuesto por las acciones que representan el $80 \%$ de la liquidez del mercado de valores peruano. Además, la cartera de referencia se estableció a partir del segundo semestre de 2012.
} 
de signo positivo (+1), y si lo es por medio de una orden de venta, será de signo negativo $(-1)$.

iii) Índice de adjudicación del precio $\left(q_{t-}\right.$ index $)$ : el índice creado es

$$
q_{t-} \text { index }=\operatorname{Ln}\left(\frac{{\text { Monto } \text { operado }_{t}}_{1 U I T}}{1 \text { II }}\right)
$$

donde el monto operado es el precio de adjudicación de la orden multiplicado por la cantidad negociada:

\section{Monto operado ${ }_{t}=P_{t}^{*}$ número de acciones transadas}

El tamaño de las transacciones está normalizado, debido a que los montos de operación son demasiado volátiles y presentan percances de revelación de información en las estimaciones. Es por ello que se evalúa si el monto efectivo marca precio o no ${ }^{18}$, siendo este el referente para las posiciones y estrategias en las operaciones realizadas en la negociación.

\section{Tratamiento econométrico}

El tratamiento econométrico aplicado en este trabajo comprende dos metodologías, un modelo MCo Newey y West (1987) y un modelo VAR (Vector Autorregresivo). En relación con la primera metodología, Harris (1986) y Hasbrouck (1991a) sostienen que los patrones de covarianza observados en los retornos de las transacciones son más consistentes con el tiempo de adjudicación de la transacción que con el tiempo "calendario"; por lo tanto, se infiere que el "tiempo" es relevante en la transacción. Dado que las variaciones pueden depender del tiempo del día, del tamaño de la operación, y de otros, es probable que los errores sean heteroscedásticos. Por otra parte, si la ecuación (21) no es exacta, el error de la regresión tiene un patrón de correlación de una serie MA $(1)^{19}$. Con esta estructura del error, el modelo MCO ofrece estimaciones puntuales y consistentes; sin embargo, la usual fórmula del error estándar es incorrecta. Por esta razón, se utiliza la metodología Newey y West (1987) con la finalidad de que la estimación de los parámetros sea consistente y que la varianza sea estimada adecuadamente.

\footnotetext{
18 Para que un valor marque precio en el mercado de acciones peruano, el monto por operación debe ser mayor o igual a una Unidad Impositiva Tributaria (UIT) (la que en 2012 fue de 3.650 nuevos soles). En el caso de que no se llegara a ese monto, el precio no variaría y seguiría siendo el precio de mercado anterior.

19 Véase Jong, Nijman y Röell (1996).
}

Sin embargo, el modelo presentado podría tener dos desventajas. En primer lugar, las estimaciones suponen una especificación correcta del modelo. Por ejemplo, se supone que toda la información asimétrica se revela inmediatamente después de la transacción, de modo que solo hay un efecto inmediato de los precios de la negociación y no hay efectos retardados. En segundo lugar, se asume que el patrón de la negociación es exógeno ${ }^{20}$. Si este patrón no fuera exógeno, los coeficientes de la regresión pudieran estar sesgados debido a que algunas variables rezagadas relevantes podrían estar omitidas.

Dadas estas consideraciones, se estima un modelo VAR, introducido en la literatura microestructural del mercado por Hasbrouck (1991a y 1993), modelo que tiene en cuenta los problemas mencionados en el párrafo anterior. En el VAR, el precio de adjudicación y la dinámica de la negociación se modelan mediante el siguiente sistema de ecuaciones:

$$
\begin{aligned}
&\left(\begin{array}{cc}
1 & -b_{0} \\
0 & I
\end{array}\right)\left(\begin{array}{c}
\Delta p_{t} \\
x_{t}
\end{array}\right)=\left(\begin{array}{ll}
a(L) & b(L) \\
c(L) & d(L)
\end{array}\right)\left(\begin{array}{c}
\Delta p_{t-i} \\
x_{t-i}
\end{array}\right)+\left(\begin{array}{l}
u_{1 t} \\
u_{2 t}
\end{array}\right), \\
& V\left(\begin{array}{l}
e_{1 t} \\
e_{2 t}
\end{array}\right)=\left(\begin{array}{cc}
\sigma^{2} & 0 \\
0 & \Omega
\end{array}\right)
\end{aligned}
$$

donde $\Delta p_{t}$ es la variación del precio y $x_{t}$ es el vector de variables explicativas, donde $a(L), b(L), c(L)$ y $d(L)$ son polinomios en el operador de rezago. En el presente análisis, el vector de variables explicativas es la dirección de la orden $\left(Q_{t}\right)$ y el tamaño del flujo de la orden para la adjudicación $\left(Z_{t}=Q_{t} q_{t}\right)$, asumiendo que los términos del error no están correlacionados. Así, este modelo permite analizar una dependencia en la variación del precio, la dirección de la orden y el tamaño del flujo de la orden respecto de las operaciones pasadas, sin el supuesto de que el patrón de la adjudicación de la orden es exógeno ${ }^{21}$.

Para analizar los shocks de los errores $\left(e_{1}, e_{2}\right)$ en los retornos futuros $(p)$ y en las variables exógenas $x_{t}=\left(Q_{t}, Z_{t}\right)$, se debe evaluar el valor esperado del precio $\tau$ períodos después del shock, dado que el sistema ha convergido a un estado estacionario ${ }^{22}$.

\footnotetext{
${ }^{20}$ Es decir, las variables explicativas no dependen de otras (que no sean ellas mismas) ni de rezagos.

21 Tal supuesto se hizo en Glosten y Harris (1988); Harris (1986); Hasbrouck (1988) y Stoll (1989).

22 Esta ecuación es para el caso de que $x_{t}$ y $e_{2 t}$ sean escalares. En el caso de un vector de negociación multidimensional, los impulsos respuestas deben ser calculados a partir de un modelo VAR con innovaciones ortogonales. En este trabajo, ello se logra mediante la adición de la variable $Q_{t}$ como una variable explicativa en la ecuación para el tamaño $Z_{t}$ a fin de obtener los errores ortogonales.
} 


$$
\begin{gathered}
p e_{1}(\tau)= \\
E\left(p_{t+\tau}-y_{t} \mid e_{1 t}=1, e_{2 t}=0, \Delta p_{t-1}=0, \ldots, x_{t-1}=0, \ldots\right) \\
p e_{2}(\tau)= \\
E\left(p_{t+\tau}-y_{t} \mid e_{1 t}=0, e_{2 t}=0, \Delta p_{t-1}=0, \ldots, x_{t-1}=0, \ldots\right)
\end{gathered}
$$

Sims (1980) popularizó la idea de calcular dichos efectos en los precios mediante las funciones de impulsorespuesta de un modelo VAR, que puede calcularse invirtiendo el VAR al siguiente vector de medias móviles (VMA):

$$
\left(\begin{array}{c}
\Delta p_{t} \\
x_{t}
\end{array}\right)=\left(\begin{array}{ll}
\alpha(L) & \beta(L) \\
\gamma(L) & \delta(L)
\end{array}\right)\left(\begin{array}{l}
e_{1 t} \\
e_{2 t}
\end{array}\right)
$$

donde $\alpha(L), \beta(L), \gamma(L)$ y $\delta(L)$ son las medias móviles de las variables antes mencionadas. Finalmente, especificando el modelo VAR bajo el enfoque microestructural se define el siguiente sistema de ecuaciones:

$$
\begin{aligned}
& \Delta p_{t}=\sum_{k=0}^{\infty} \alpha_{k} e_{1, t-k}+\sum_{k=0}^{\infty} \beta_{k} e_{2, t-k} \\
& \Delta x_{t}=\sum_{k=0}^{\infty} \gamma_{k} e_{1, t-k}+\sum_{k=0}^{\infty} \delta_{k} e_{2, t-k}
\end{aligned}
$$

Cabe notar, por ejemplo, que en (28) las diferencias de precios son sumas infinitas de los shocks en los retornos futuros y en las variables exógenas. Asimismo, en la ecuación antes mencionada, el efecto del precio y las innovaciones de las variables de negociación $\left(Q_{t} \mathrm{y}\right.$ $Z_{t}$ ) en el precio se miden por las respuestas de impulsos $\alpha_{k}$ y $\beta_{k}$, respectivamente. Por lo tanto, los efectos de un shock en el nivel de precios ( $\tau$ períodos adelante) se miden por las sumas parciales de las respuestas de impulso:

$$
p e_{1}(\tau)=\sum_{k=0}^{\tau} \alpha_{k} \text { y } p e_{2}(\tau)=\sum_{k=0}^{\tau} \beta_{k}
$$

Los efectos de largo plazo de los shocks serían los límites de las sumas parciales de $\tau \rightarrow \infty$ :

$$
\begin{gathered}
p e_{1}(\infty)=\sum_{k=0}^{\infty} \alpha_{k}=\alpha(1) \quad \mathrm{y} \\
p e_{2}(\infty)=\sum_{k=0}^{\infty} \beta_{k}=\beta(1)
\end{gathered}
$$

Dado lo anterior, si los impulsos-respuestas dados por los shocks se disipan en el tiempo, se infiere que los shocks de iliquidez son meramente temporales. En cambio, si estos no se diluyen, se concluye que son permanentes y poseen un efecto en el largo plazo. En este sentido, Cochrane (1988) señala que esta definición de los efectos de largo plazo de las innovaciones es única e independiente de cualquier descomposición particular del proceso de adjudicación de precios para los efectos transitorios y permanentes.

Finalmente, en cuanto al uso del "signo" para la dirección de la orden en un modelo dinámico simultáneo, este genera algunos problemas para la estimación y la computación de efectos dinámicos. Debido a que $Q_{t}$ es una variable dependiente limitada que solo puede tomar los valores $-1 \mathrm{y}+1$, la primera ecuación del VAR no puede ser una expectativa condicional de $Q_{t}$ para todos los valores de $\Delta p_{t-\mathrm{i}}$ IR si los coeficientes de $\Delta p_{t-\mathrm{i}}$ no son cero. Sin embargo, para valores moderados de $\Delta p_{t-\mathrm{i}}$ la ecuación lineal puede ser una buena aproximación de la verdadera esperanza condicional ${ }^{23}$. Por consiguiente, el uso de $Q_{t}$ como una variable explicativa en la ecuación $\Delta p_{t}$ no genera problemas, ya que los errores de la ecuación del retorno y las otras ecuaciones del VAR no están correlacionados ${ }^{24}$. Se proponen cinco rezagos ${ }^{25}$ en el VAR como suficientes, dada la ausencia general de correlación serial residual en las ecuaciones estimadas, siendo los errores estándar estimaciones consistentes de heterocedasticidad.

\section{Resultados de la estimación}

La primera ecuación estimada es la (21), que representa la dinámica de precios y se vuelve a presentar en esta sección para facilitar la lectura de los resultados.

$$
\begin{aligned}
\Delta p_{t}=c+ & R_{0} \Delta Q_{t-1}+R_{1} \Delta\left(q_{t-1} Q_{t-1}\right) \\
& +g_{0} Q_{t}+g_{1} q_{t} Q_{t} e_{t}
\end{aligned}
$$

La hipótesis que se contrasta es que la variación del precio depende de la dirección de la orden $\left(Q_{t}\right)$ y del volumen negociado $\left(q_{t}\right)$. Los resultados se presentan en dos cuadros: en el cuadro 1 se muestran los resultados para las cinco acciones más líquidas del IGBVL, y el cuadro 2 corresponde a las cinco acciones menos líquidas del IGBVL. En el cuadro 1 se puede observar,

\footnotetext{
23 Véase Jong, Nijman y Röell (1996).

24 Véase Heckman (1978).

25 Véase Hasbrouck (1991b).
} 
para las cinco acciones, que los coeficientes de dirección de la orden $R_{0}$ y $g_{0}$ tienen signos positivo y negativo, respectivamente. Sin embargo, $R_{1}$ y $g_{1}$ (debido a RIO y CVERDEC1) no cumplen con tener siempre signos negativo y positivo, respectivamente. La regresión de la acción Cerro Verde solo difiere en el signo del parámetro $R_{1}$ y la que corresponde a la acción Rio Alto, en el signo del parámetro $g_{1}$. En el cuadro 2, las cinco regresiones presentan coeficientes con los mismos signos expresados en los resultados del cuadro 1 , sin presentar excepciones.

En el cuadro 1 se muestran los coeficientes estimados del modelo de Jong, Nijman y Röell (1996) a través de la ecuación (21), siendo las variaciones de los precios en centésimas de punto porcentual, y las cantidades denominadas en el índice de adjudicación de precios $\left(q_{t-}\right.$ index). Los valores escogidos son las acciones más líquidas, las primeras cinco acciones del IGBVL del año
2012. Asimismo, $\alpha$ es estimado por la mediana de la distribución del $q_{t-}$ index, dividido entre 2 , y $\rho$ es el coeficiente de autocorrelación de primer orden del error. Los valores $p$, corregidos por la metodología NeweyWest, aparecen entre corchetes.

En el cuadro 2 aparecen los coeficientes estimados del modelo de Jong, Nijman y Röell (1996), a través de la ecuación (21), midiendo las variaciones de los precios en centésimas de punto porcentual, y utilizando el índice de adjudicación de precios $\left(q_{t-}\right.$ index $)$ como proxy del número de acciones transadas. Los valores escogidos son las acciones menos líquidas, las primeras cinco acciones del IGBVL del año 2012. Asimismo, $\alpha$ es estimado por la mediana de la distribución del $q_{t-}$ index, dividido entre 2 , y $\rho$ es el coeficiente de autocorrelación de primer orden del error. Los valores $p$, corregidos por la metodología Newey-West, se denotan entre corchetes.

CUADRO 1

Coeficientes estimados de la adjudicación de precios para las cinco acciones más líquidas del IGBVL, 2012

\begin{tabular}{|c|c|c|c|c|c|c|c|c|c|}
\hline Valor & Nombre de la acción & $R_{0}$ & $R_{1}$ & $g_{0}$ & $g_{1}$ & $\alpha$ & $\rho$ & $C_{0}$ & $C_{1}$ \\
\hline Volcan "B" & VOLCABC1 & $\begin{array}{c}2,074 \\
{[0,000]}\end{array}$ & $\begin{array}{l}-0,281 \\
{[0,000]}\end{array}$ & $\begin{array}{l}-5,434 \\
{[0,000]}\end{array}$ & $\begin{array}{c}0,666 \\
{[0,000]}\end{array}$ & 0,732 & $-0,046$ & 7,020 & $-0,614$ \\
\hline Rio Alto Mining & RIO & $\begin{array}{c}1,597 \\
{[0,000]}\end{array}$ & $\begin{array}{l}-0,044 \\
{[0,757]}\end{array}$ & $\begin{array}{l}-7,400 \\
{[0,000]}\end{array}$ & $\begin{array}{l}-0,372 \\
{[0,019]}\end{array}$ & 0,686 & $-0,172$ & 9,252 & 0,142 \\
\hline FerreyCorp & FERREYC1 & $\begin{array}{c}1,749 \\
{[0,000]}\end{array}$ & $\begin{array}{l}-0,108 \\
{[0,426]}\end{array}$ & $\begin{array}{l}-6,901 \\
{[0,000]}\end{array}$ & $\begin{array}{c}0,558 \\
{[0,001]}\end{array}$ & 0,716 & $-0,051$ & 8,250 & $-0,387$ \\
\hline Cerro Verde & CVERDEC1 & $\begin{array}{c}0,809 \\
{[0,113]}\end{array}$ & $\begin{array}{c}0,025 \\
{[0,928]}\end{array}$ & $\begin{array}{l}-5,792 \\
{[0,000]}\end{array}$ & $\begin{array}{c}0,524 \\
{[0,013]}\end{array}$ & 0,973 & $-0,072$ & 6,091 & $-0,238$ \\
\hline ADR Buenaventura & BVN & $\begin{array}{c}1,246 \\
{[0,354]}\end{array}$ & $\begin{array}{l}-0,765 \\
{[0,096]}\end{array}$ & $\begin{array}{c}-15,832 \\
{[0,000]}\end{array}$ & $\begin{array}{c}3,408 \\
{[0,000]}\end{array}$ & 0,868 & $-0,234$ & 14,119 & $-2,469$ \\
\hline
\end{tabular}

Fuente: elaboración propia.

CUADRO 2

Coeficientes estimados de la adjudicación de precios para las cinco acciones menos líquidas del IGBVL, 2012

\begin{tabular}{|c|c|c|c|c|c|c|c|c|c|}
\hline Valor & Nombre de la acción & $R_{0}$ & $R_{1}$ & $g_{0}$ & $g_{1}$ & $\alpha$ & $\rho$ & $C_{0}$ & $C_{1}$ \\
\hline Austral Group & AUSTRAC1 & $\begin{array}{l}27,760 \\
{[0,000]}\end{array}$ & $\begin{array}{l}-4,687 \\
{[0,000]}\end{array}$ & $\begin{array}{c}-48,051 \\
{[0,000]}\end{array}$ & $\begin{array}{c}8,887 \\
{[0,000]}\end{array}$ & 0,474 & $-0,180$ & 71,602 & $-9,131$ \\
\hline El Brocal & BROCALC1 & $\begin{array}{c}2,789 \\
{[0,017]}\end{array}$ & $\begin{array}{l}-0,357 \\
{[0,524]}\end{array}$ & $\begin{array}{c}-10,243 \\
{[0,000]}\end{array}$ & $\begin{array}{c}0,109 \\
{[0,900]}\end{array}$ & 0,492 & $-0,060$ & 12,978 & $-0,411$ \\
\hline $\begin{array}{l}\text { Agro Industria } \\
\text { Pomalca }\end{array}$ & POMALCC1 & $\begin{array}{l}13,416 \\
{[0,000]} \\
\end{array}$ & $\begin{array}{l}-0,930 \\
{[0,666]}\end{array}$ & $\begin{array}{c}-42,864 \\
{[0,000]} \\
\end{array}$ & $\begin{array}{c}4,989 \\
{[0,054]} \\
\end{array}$ & 0,075 & $-0,235$ & 55,905 & $-3,425$ \\
\hline EDELNOR & EDELNOC1 & $\begin{array}{c}1,257 \\
{[0,359]} \\
\end{array}$ & $\begin{array}{l}-0,521 \\
{[0,490]}\end{array}$ & $\begin{array}{l}-7,083 \\
{[0,000]}\end{array}$ & $\begin{array}{c}0,960 \\
{[0,200]}\end{array}$ & 0,810 & $-0,225$ & 7,562 & $-1,001$ \\
\hline Scotiabank & SCOTIAC1 & $\begin{array}{c}6,836 \\
{[0,025]}\end{array}$ & $\begin{array}{l}-2,750 \\
{[0,010]}\end{array}$ & $\begin{array}{c}-16,164 \\
{[0,000]}\end{array}$ & $\begin{array}{c}4,613 \\
{[0,002]}\end{array}$ & 0,546 & $-0,372$ & 20,482 & $-5,056$ \\
\hline
\end{tabular}

Fuente: elaboración propia. 
El signo positivo de $R_{0}$ significa que el mercado ingresa órdenes consecutivas con la misma dirección y ello determina que la variación sea positiva o negativa; si la operación se inicia como compra tenderá a "mejorar el precio" 26 o a subirlo (ya que en el LOB comenzarán a existir más propuestas de compra y aumentará la demanda); por el contrario, si inicia como venta tenderá a disminuirlo (ya que en el LOB comenzarán a existir más propuestas de venta y la oferta aumentará). El signo negativo de $R_{1}$ se interpreta como la contraposición para el cierre de la operación. En este caso se debe recordar que el modelo está formulado desde el lado del comprador; siendo así, para que una orden se ejecute debe tener una operación opuesta a la suya, de modo que si un inversionista realiza una orden de compra (signo positivo), esta debe ser asignada a una orden de venta (signo negativo). Los coeficientes $g_{0}$ y $g_{1}$ se interpretan con la misma lógica que los coeficientes en "variaciones", con la única diferencia de que los primeros capturan la dinámica de las ejecuciones pasadas, en cambio los de niveles reflejan el costo de la adjudicación actual.

En relación con la magnitud de los coeficientes estimados, en ambos cuadros se puede observar que los coeficientes correspondientes a la dirección de las órdenes $\left(R_{0}\right.$ y $\left.g_{0}\right)$ son mayores que los relacionados con el volumen de acciones $\left(R_{1}\right.$ y $\left.g_{1}\right)$. Sin embargo, se aprecia que las acciones más líquidas (cuadro 1 ), en promedio, tienen coeficientes de dirección de la orden y el volumen de acciones de mayor magnitud absoluta con respecto a los coeficientes de las acciones menos líquidas (cuadro 2). La interpretación natural de esta diferencia es que las variaciones de los precios de las acciones menos líquidas son más sensibles a la dirección de la orden y al volumen de acciones.

En los cuadros 3 y 4 se señala la composición del costo que asumen los inversionistas por ejecutar una orden, según el modelo de Glosten (1994) extendido por Jong, Nijman y Röell (1996). Este costo está compuesto por el costo de selección adversa, calculado a partir de la ecuación (11) y los costos de procesamiento, calculados

\footnotetext{
${ }^{26}$ Para que un precio "mejore" se debe tener en cuenta quién inicia la operación. En caso de que sea la compra, el precio mejora si la variación es positiva. Por el contrario, si la operación es iniciada por la venta, el precio mejora si la variación es negativa. En ambos casos, el precio mejora porque obliga a aumentar o disminuir los límites de las propuestas (dependiendo del lado de la compra o de la venta), reduciendo el spread.
}

a partir de la ecuación (12). Siendo las cantidades denominadas con respecto al índice de adjudicación de precios $\left(q_{t-}\right.$ index $)$. Por ejemplo, para el caso de la acción más líquida del IGBVL, VOLCABC1, el mínimo número de acciones que comprar de esta empresa para generar beneficios por comprar sería igual a 6.224 acciones. Donde la operación generaría un costo total de S/. 18.671, siendo este el monto negociado, en otras palabras, el precio de la acción multiplicado por el número de acciones transadas. Respecto de la composición del costo anterior, el 57,76\% correspondería al costo de procesamiento de la orden (S/. 10.784), y el $42,24 \%$ al costo de selección adversa (S/. 7.887). Por otra parte, en el caso de la acción menos líquida del IGBVL, SCOTIAC1, el mínimo número de acciones por comprar de esta empresa para generar beneficios positivos sería igual a 595 acciones. Tal compra generaría un costo total de S/. 22.905, donde el costo del procesamiento de la orden correspondería al 54,40\% (S/. 12.460) y el costo de selección adversa al 45,60\% (S/. 10.445).

Los valores absolutos de ambos costos son mayores en el caso de las acciones menos líquidas con respecto a las más líquidas; de modo que el volumen óptimo de negociación de acciones para obtener beneficios iguales a cero es mayor para el caso de las acciones menos líquidas. En relación a la posición relativa de cada costo respecto al costo total, se observa que tanto las acciones más líquidas y menos líquidas, la proporción correspondiente al costos de procesamiento es mayor que al costo de selección adversa.

En el cuadro 3 se revela desde qué punto los beneficios son positivos, mayores o iguales a cero, al operar (en el año 2012) en el mercado, dependiendo de cada tipo de acción. Los valores escogidos son las acciones más líquidas, las primeras cinco acciones del IGBVL del año 2012. Para pasar de monto operado a número de acciones transadas se utiliza como precio de referencia la mediana del logaritmo natural del precio.

En el cuadro 4 se evidencia desde qué punto los beneficios son positivos, mayores o iguales a cero, al operar (en 2012) en el mercado dependiendo de cada tipo de acción. Siendo las cantidades denominadas con respecto al índice de adjudicación de precios $\left(q_{t-}\right.$ index $)$. Los valores escogidos son las acciones menos líquidas, las últimas cinco acciones, del IGBVL del año 2012. Para pasar de monto operado a número de acciones transadas, se utiliza como precio de referencia la mediana del logaritmo natural del precio. 
CUADRO 3

Composición del costo de adjudicación de órdenes para las cinco acciones más líquidas del IGBVL, 2012

\begin{tabular}{|c|c|c|c|c|c|}
\hline & VOLCABC1 & RIO & FERREYC1 & CVERDEC1 & BVN \\
\hline$\hat{q}_{t-i}$ index & 1,6186 & 1,5290 & 1,5783 & 0,8290 & 0,7059 \\
\hline Monto operado (en S/.) & 18671 & 17070 & 17933 & 8476 & 7495 \\
\hline Número de acciones transadas & 6224 & 3924 & 7599 & 215 & 192 \\
\hline Costo de procesamiento (normalizado) & 6,02 & 9,47 & 7,64 & 5,89 & 12,38 \\
\hline (en términos de $q_{t-}$ index, y en valores absolutos) & $57,76 \%$ & $54,39 \%$ & $55,76 \%$ & $53,78 \%$ & $51,47 \%$ \\
\hline Costo de selección adversa & 4,41 & 7,94 & 6,06 & 5,06 & 11,67 \\
\hline (en términos de $q_{t-}$ index, y en valores absolutos) & $42,24 \%$ & $45,61 \%$ & $44,24 \%$ & $46,22 \%$ & $48,53 \%$ \\
\hline
\end{tabular}

Fuente: elaboración propia.

CUADRO 4

Composición del costo de adjudicación de órdenes para las cinco acciones menos líquidas del IGBVL, 2012

\begin{tabular}{|c|c|c|c|c|c|}
\hline & AUSTRAC1 & BROCALC1 & POMALCC1 & EDELNOC1 & SCOTIAC 1 \\
\hline$\hat{q}_{t-}$ index & 4,8810 & 2,0560 & 6,9510 & 0,8266 & 1,8230 \\
\hline Monto operado (en S/.) & 487539 & 28916 & 3863510 & 8457 & 2905 \\
\hline Número de acciones transadas & 1875151 & 629 & 7289639 & 2135 & 595 \\
\hline Costo de procesamiento (normalizado) & 27,03 & 12,13 & 32,10 & 6,73 & 11,26 \\
\hline (en términos de $q_{t-}$ index, y en valores absolutos) & $54,96 \%$ & $54,63 \%$ & $56,07 \%$ & $53,27 \%$ & $54,40 \%$ \\
\hline Costo de selección adversa & 22,15 & 10,08 & 25,15 & 5,91 & 9,44 \\
\hline (en términos de $q_{t-}$ index, y en valores absolutos) & $45,04 \%$ & $45,37 \%$ & $43,93 \%$ & $46,73 \%$ & $45,60 \%$ \\
\hline
\end{tabular}

Fuente: elaboración propia.

Por otra parte, los costos de procesamiento y de selección adversa cambian según aumenta o disminuye el índice del número de acciones negociadas. Este ejercicio se ha reproducido para las ocho acciones restantes, y se puede encontrar en el anexo A. A continuación se muestra el caso de dos acciones, VOLCABC1 (gráfico 1) y POMALCC1 (gráfico 2), que ilustran las diferencias en el comportamiento entre una acción del grupo de acciones más líquida y una acción del grupo de acciones menos líquidas. Puede notarse que en el caso de volCABC1, los costos de procesamiento disminuyen relativamente y se incrementan los costos de selección adversa, un resultado muy diferente al caso de POMALCC1, donde la proporción de cada costo prácticamente permanece.

En gráfico 1 se advierte el comportamiento de los componentes de los costos de la acción VOLCABC1, perteneciente al grupo de acciones más líquidas del IGBVL. Se puede observar que conforme se incrementa el índice de número de acciones negociadas el costo de procesamiento desciende $\mathrm{y}$, por lo tanto, el costo de selección adversa aumenta.

En el gráfico 2 se aprecia el comportamiento de los componentes de los costos de la acción POMALCC1, perteneciente al grupo de acciones menos líquidas del IGBVL. Se puede observar que conforme se incrementa el índice de número de acciones negociadas, el costo de procesamiento desciende mínimamente y, por lo tanto, el costo de selección adversa aumenta lentamente.

En relación con los componentes del spread, el modelo teórico predice que cuando aumenten los montos negociados, el costo de procesamiento de la orden disminuirá y el costo por selección adversa aumentará. Este comportamiento se espera debido a que al operar montos pequeños, que no marcan precio y que en términos del $q_{t}$ index son de ]- $\infty, 0[$, no afecta al mercado ni a las futuras posiciones en las expectativas de este, por lo que el costo más relevante es el costo (de procesamiento) implícito por operar. Por el contrario, cuando se operan montos mayores la información se vuelve relevante, y los montos de negociación son indicadores que entregan información sobre las posiciones en el activo. En esta situación, los inversionistas desearán tomar una posición o adjudicar un precio, dado que posiciones significativas dentro de una cartera crean una alta exposición del portafolio a variaciones en los respectivos precios. 
GRÁFICO 1

Comportamiento del costo de negociación de volCABC1

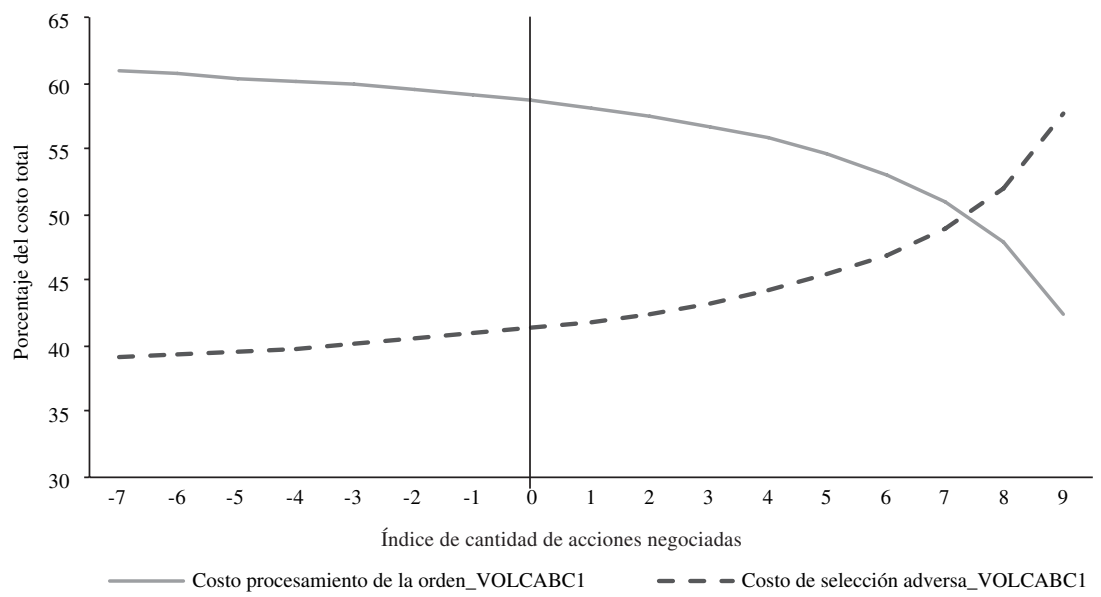

Fuente: elaboración propia.

GRÁFICO 2

Comportamiento del costo de negociación de POMALCC1

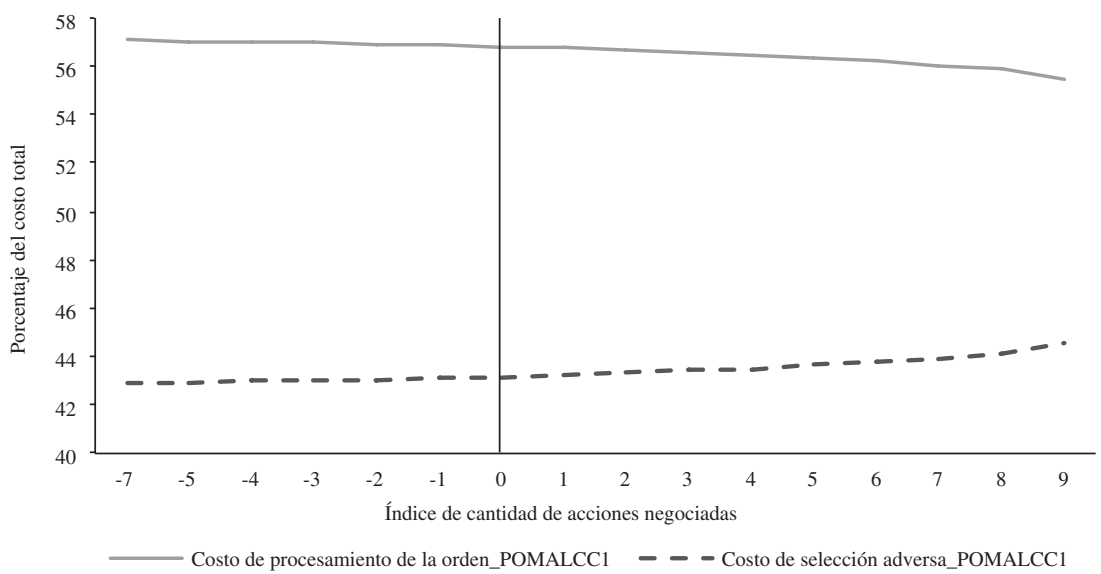

Fuente: elaboración propia.

El efecto en la variación del precio causado por la dirección de la orden y del volumen puede evaluarse en los subsiguientes períodos después del efecto en el tiempo $t$, esto con la finalidad de analizar su permanencia en las subsiguientes operaciones. Los resultados revelan que, en el grupo de acciones más líquidas, la permanencia del efecto en el precio es menor con respecto al grupo de acciones menos líquidas. Este análisis puede encontrarse en el anexo B. A continuación, se presenta el análisis para VOLCABC1 en el gráfico 3 y para POMALCC1 en el gráfico 4, donde puede evidenciarse el resultado expuesto anteriormente.

En los gráficos 3 y 4 se muestran los resultados del modelo VAR con respecto a la dinámica de precios
$\left(\Delta p_{t}=\mathrm{D}_{\mathrm{PT}_{\mathrm{ACCIÓ}}}\right.$, donde ACCIón toma el nombre del respectivo nemónico), la dirección de la orden ( $Q_{t}=$ SIGNO), y el tamaño del flujo de la orden para la adjudicación $\left(q_{t} Q_{t}=\right.$ SIGNO_QT_INDEX). Se encuentra que los shocks en las variables son corregidos en todas las acciones analizadas. Si bien todas las acciones muestran diferentes magnitudes en sus impulsos, se puede notar que mientras más líquida es la acción, más rápido es la corrección del mercado.

En relación con el análisis de la dinámica de la dirección de la orden y del monto operado ante un shock, en promedio el mercado ingresa órdenes con la misma dirección para luego corregirse. En caso de que la variación sea positiva (negativa), los agentes impacientes 
ingresan propuestas de compra (venta) con la expectativa de que el valor siga incrementándose (bajando). Para que el mercado se limpie, entiéndase como diluir el shock, los ofertantes (demandantes) aprovechan este rush (down) y se deshacen (o adquieren) de sus posiciones, probablemente para realizar operaciones con lógica de toma de ganancias diarias o day-trade ${ }^{27}$. Es por ello

27 Una operación day-trade tiene lugar cuando al comienzo de la negociación se compra barato, y al final de la negociación se venden las misma acciones a un precio mayor, obteniendo un beneficio. que, dependiendo del estado de la liquidez del activo, como un indicador de si el mercado se está secando ${ }^{28}$ o no, la contraparte "pegará" o adjudicará al mejor bid o ask, siendo primordiales el nivel del spread y la profundidad del LOB.

\footnotetext{
28 Que el mercado se "seque" significa que la demanda por el activo, o la oferta de este, comienza a disminuir. Por lo que el inversionista no encuentra propuestas (en dirección opuesta) para concretar la operación.
}

GRÁFICO 3

Funciones de impulso-respuesta del modelo econométrico VAR para VOLCABC1

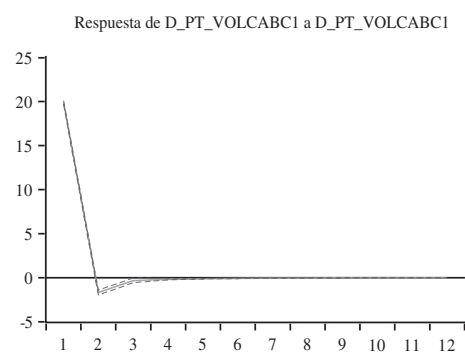

Respuesta de SIGNO a D_PT_VOLCABC1

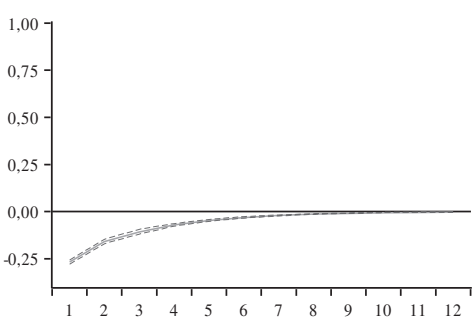

Respuesta de SIGNO_QT_INDEX a D_PT_VOLCABC1

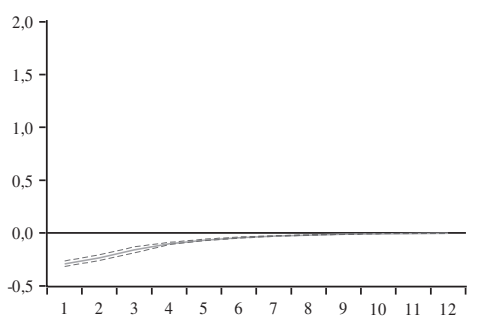

Respuesta de D PT VOLCABCl a SIGNO

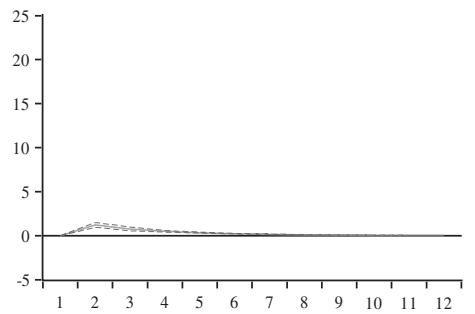

Respuesta de SIGNO a SIGNO

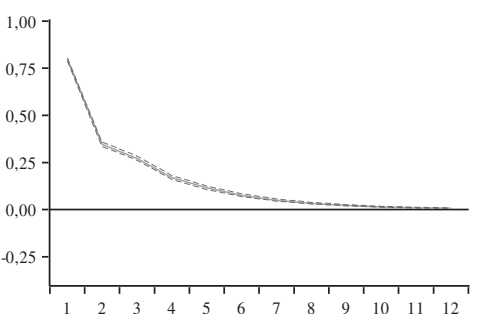

Respuesta de SIGNO_QT_INDEX a SIGNO

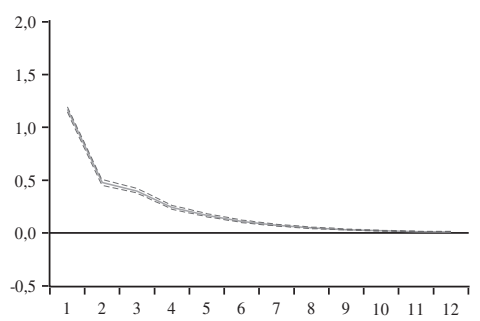

Respuesta de D PT VOLCABC1 a SIGNO OT INDEX

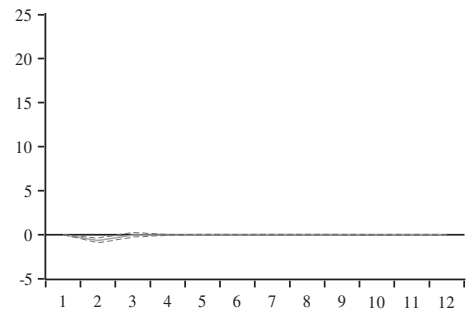

Respuesta de SIGNO a SIGNO_QT_INDEX

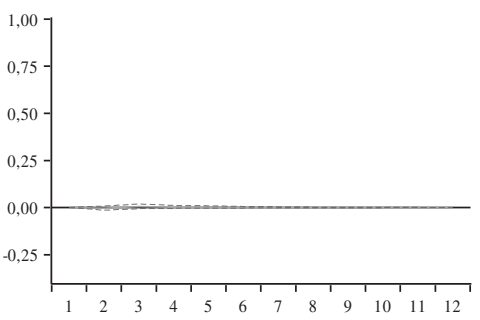

Respuesta de SIGNO_QT_INDEX a SIGNO_QT_INDEX

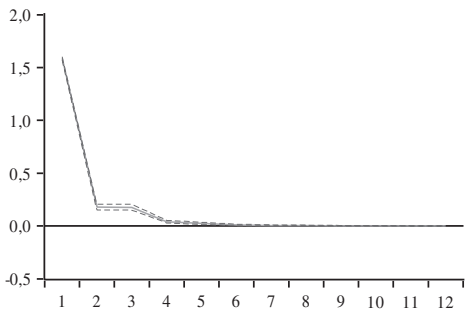

Fuente: elaboración propia. 
GRÁFICO 4

Funciones de impulso-respuesta del modelo econométrico VAR para POMALCC1
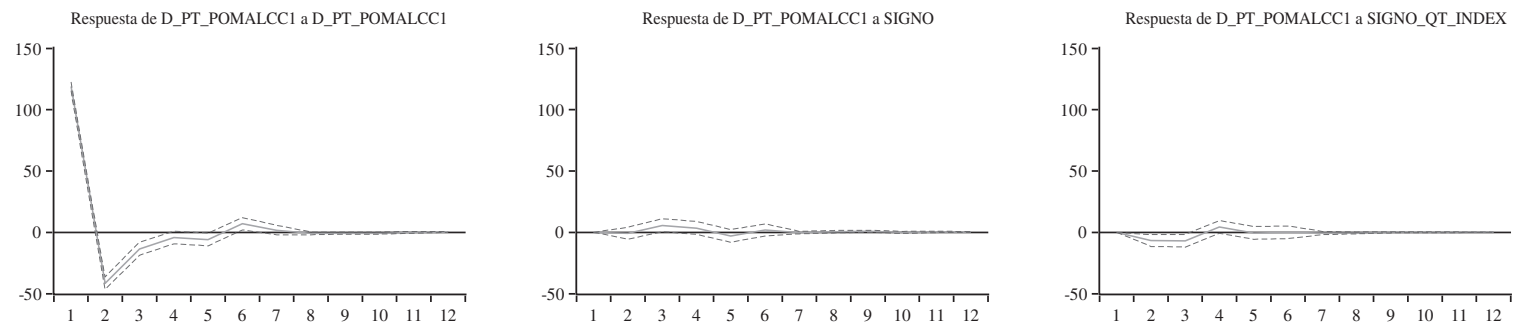

Respuesta de SIGNO a D_PT_POMALCC
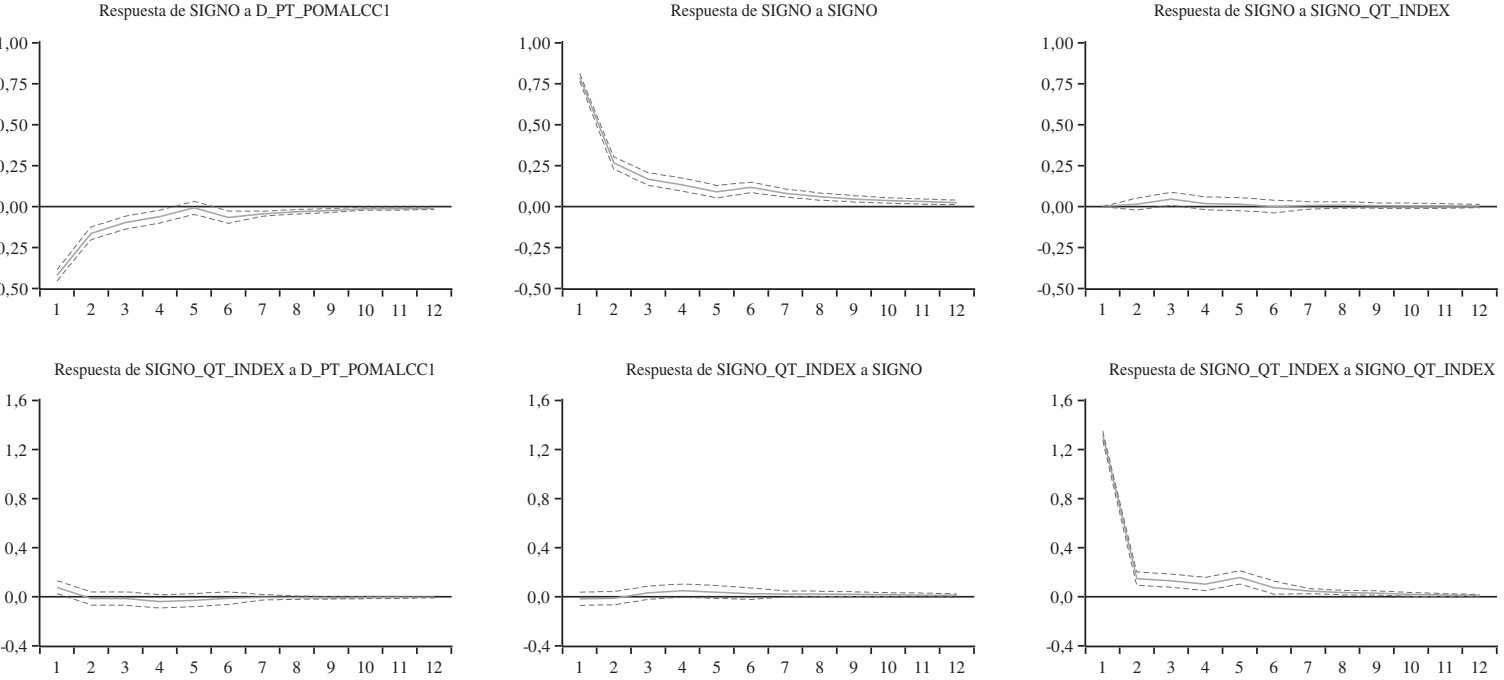

Fuente: elaboración propia.

\section{V}

\section{Conclusiones}

Los resultados empíricos, mediante la aplicación de la metodología Newey y West (1987), dejan ver que la formación de adjudicación de precios en la BVL depende de la dirección y del número o volumen de las acciones negociadas. El efecto más relevante en el precio es el de la dirección de la orden. El grupo de acciones menos líquidas presenta una mayor sensibilidad a estas variables exógenas en relación con el grupo de acciones más líquidas. Luego, los resultados obtenidos con la metodología VAR, en el análisis del efecto en el precio en los períodos posteriores, ponen de manifiesto que dicho efecto está presente en un mayor número de períodos en el caso de las acciones menos líquidas. Con lo cual, en términos de Black (1971), la resilencia es menor.

Con respecto a los costos de ejecutar una operación (costo que asume el inversionista para operar en la BVL), y a partir de los parámetros estimados con la metodología Newey y West (1987), se encuentra que para la ejecución de operaciones de acciones menos líquidas dichos costos son los más elevados. Por otra parte, los costos de selección adversa (producto de la información asimétrica en la BVL) se pueden interpretar como un mejor uso de la nueva información que llega al mercado (Stoll, 2000) a través de inversionistas especializados, 
quienes obtienen beneficios a costa de inversionistas no especializados, es decir, de aquellos que no usan la nueva información oportuna y correctamente.

En la composición de tales costos predomina el costo de procesamiento con una participación aproximada del $55 \%$, y la diferencia (45\%) corresponde a los costos de selección adversa. Cuando la composición de dichos costos se evalúa a mayores niveles de volúmenes de acciones negociados, se puede notar que la composición cambia. En el caso de la acción más líquida de la BVL, VOLCABC1, los costos de selección adversa comienzan a presentar una mayor participación. El caso es muy distinto si se trata de POMALCC1, en la que la composición no cambia. En el anexo A puede observarse que, en general, los resultados son muy distintos para cada acción.

La consecuencia en el precio de las acciones de estos costos de procesamiento y de selección adversa es que modifican las funciones de distribución de los precios adjudicados de las acciones, ensanchando o reduciendo las colas de la distribución de los precios. Mayores costos provocan funciones con colas más anchas, y por lo tanto, spreads mayores. Así, las acciones menos líquidas presentan mayores spreads que las acciones más líquidas.

Por último, utilizando los parámetros estimados con la metodología Newey y West (1987), se ha realizado un análisis adicional respecto del cálculo del mínimo de acciones por negociar para diluir los costos de procesamiento y selección adversa. Las acciones menos líquidas requieren de la negociación de un número mayor de estas para diluir los costos definidos en la teoría presentada. De este resultado se puede inferir que la formación de portafolios eficientes, con acciones menos líquidas, requiere una mayor liquidez (efectivo), dado que para alcanzar el punto de equilibrio es necesario negociar un mayor número de acciones. Esta situación podría estar contribuyendo a la baja liquidez que presenta el mercado de acciones de la BVL.

ANEXO A

En el gráfico A.1 se muestra el comportamiento de los componentes de los costos de la acción de RIO. Puede observarse que, conforme se incrementa el índice del número de acciones negociadas, el costo de procesamiento desciende mínimamente y, por lo tanto, el costo de selección adversa aumenta lentamente.

GRÁFICO A.1

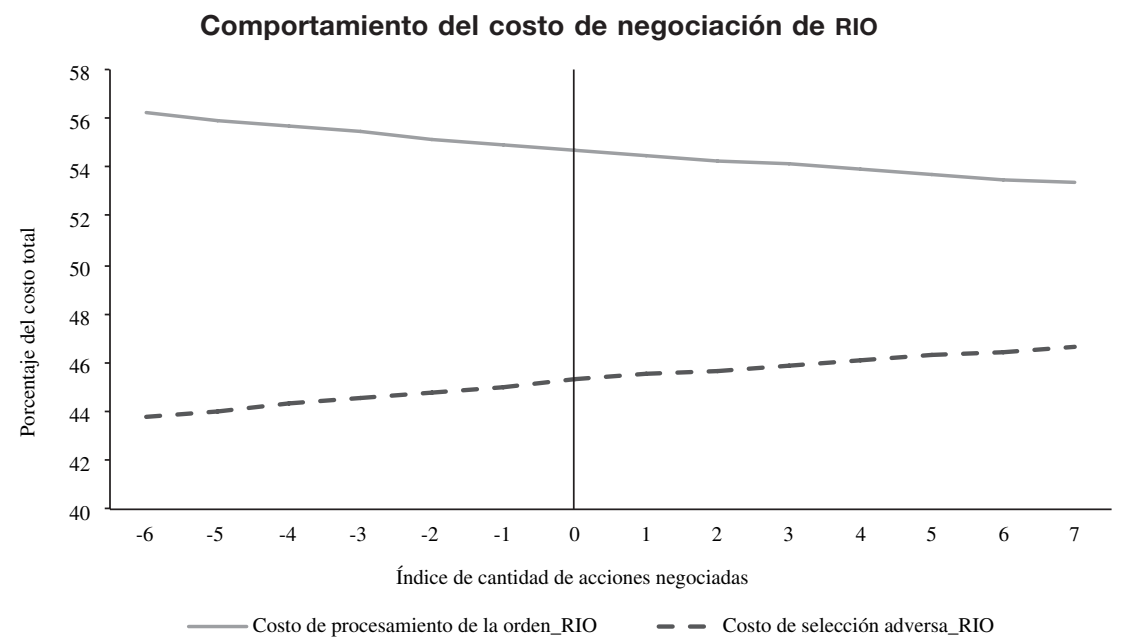

Fuente: elaboración propia. 
En el gráfico A.2 se aprecia el comportamiento de los componentes de los costos de la acción de FERREYC1. Puede observarse que, conforme se incrementa el índice del número de acciones negociadas, el costo de procesamiento desciende mínimamente y, por lo tanto, el costo de selección adversa aumenta lentamente.

GRÁFICO A.2

\section{Comportamiento del costo de negociación de FERREYC1}

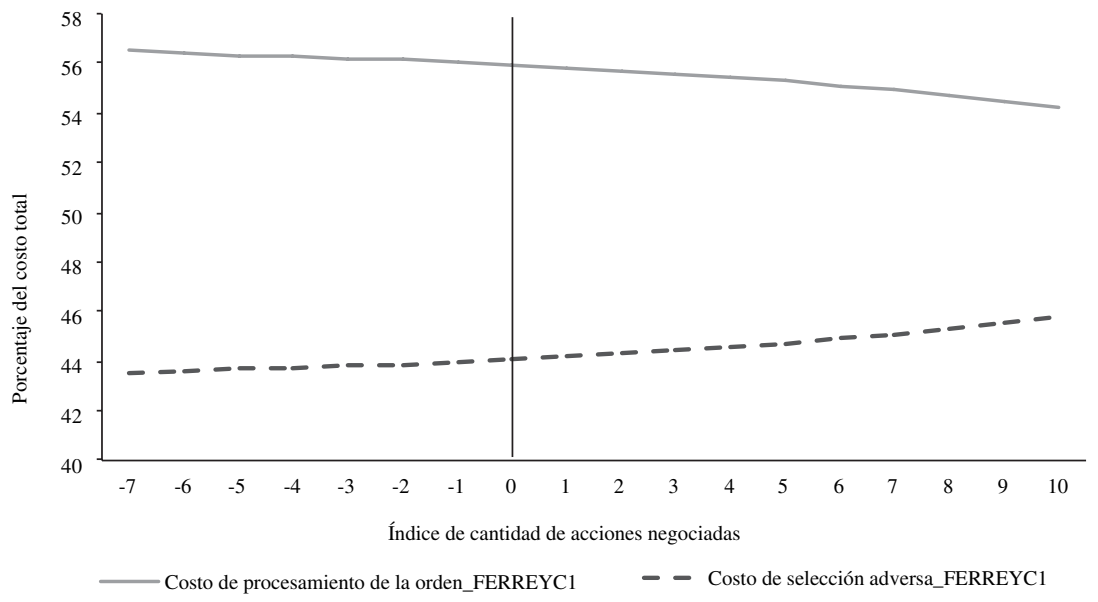

Fuente: elaboración propia.

En el gráfico A.3 se expresa el comportamiento de los componentes de los costos de la acción de CVERDEC1. Puede observarse que, conforme se incrementa el índice del número de acciones negociadas, el costo de procesamiento asciende mínimamente y, por lo tanto, el costo de selección adversa disminuye.

GRÁFICO A.3

Comportamiento del costo de negociación de CVERDEC1

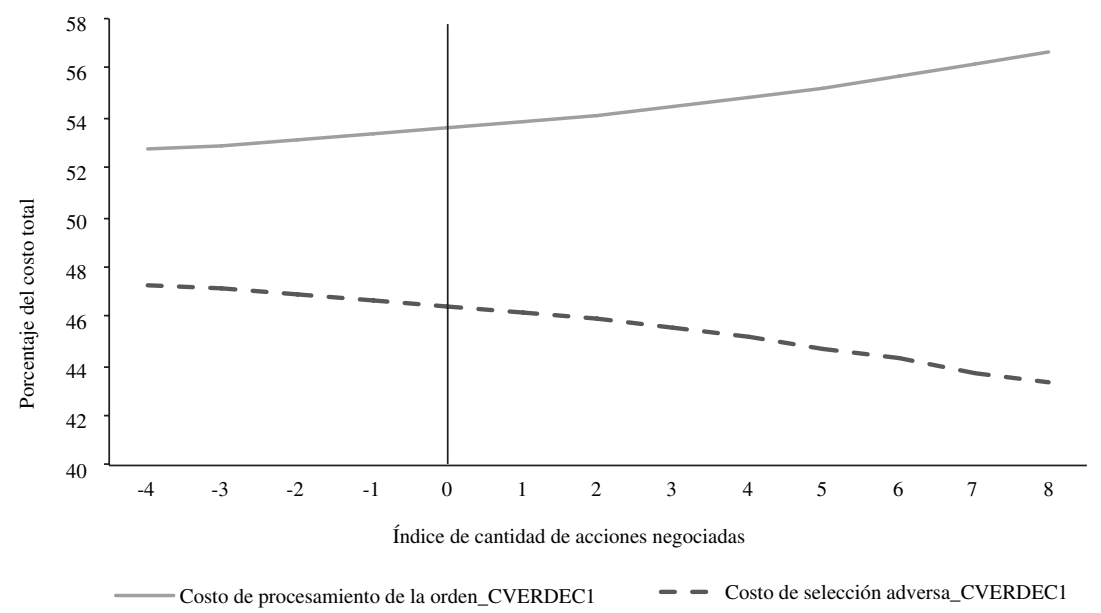

Fuente: elaboración propia 
En el gráfico A.4 se evidencia el comportamiento de los componentes de los costos de la acción de BVN. Puede observarse que, conforme se incrementa el índice del número de acciones negociadas, el costo de procesamiento desciende rápidamente y, por lo tanto, el costo de selección adversa aumenta. Un aspecto curioso es que alcanza un nivel en que se revierte la tendencia, por lo que sería interesante modelar estos componentes bajo una metodología no lineal.

GRÁFICO A.4

\section{Comportamiento del costo de negociación de BVN}

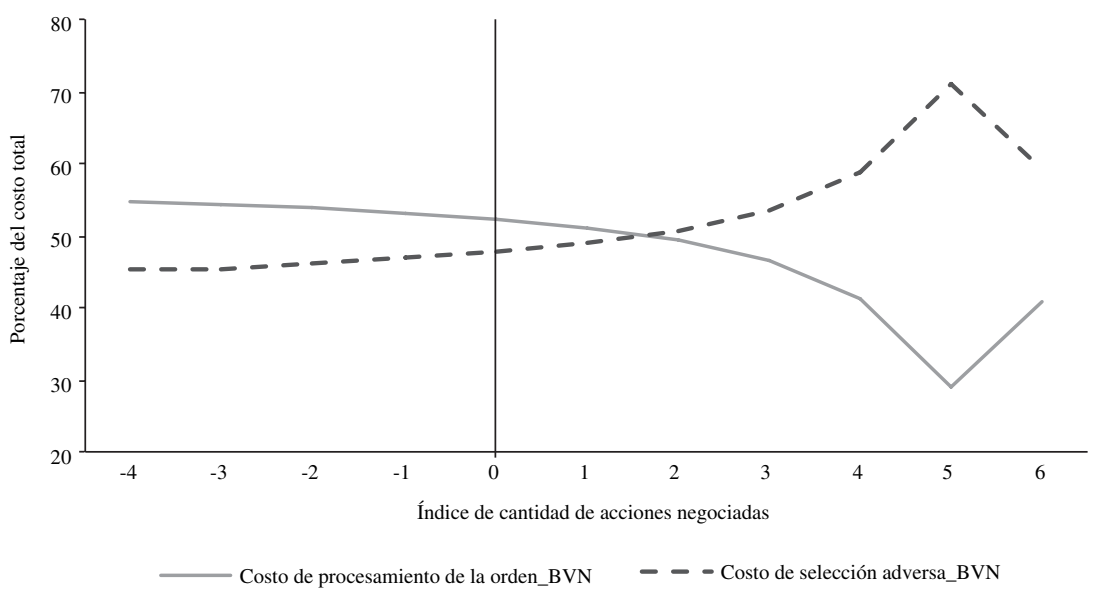

Fuente: elaboración propia.

En el gráfico A.5 se advierte el comportamiento de los componentes de los costos de la acción de BROCALC1. Puede observarse que, conforme se incrementa el índice del número de acciones negociadas, el costo de procesamiento desciende rápidamente $\mathrm{y}$, por lo tanto, el costo de selección adversa aumenta.

GRÁFICO A.5

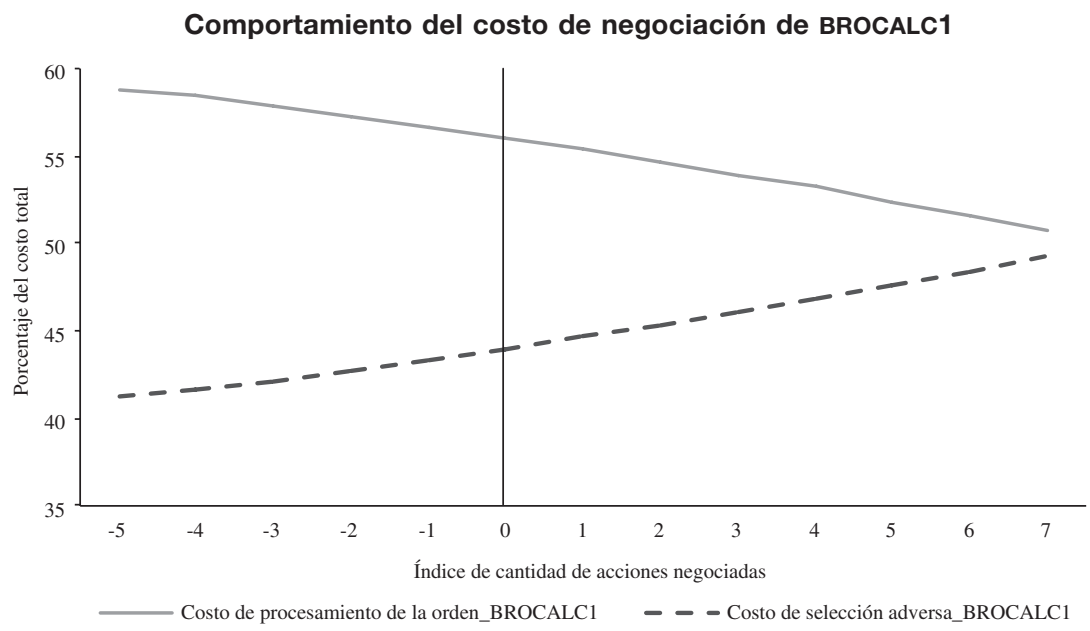

Fuente: elaboración propia. 
En el gráfico A.6 se aprecia el comportamiento de los componentes de los costos de la acción de AUSTRAC1. Puede observarse que, conforme se incrementa el índice del número de acciones negociadas, el costo de procesamiento desciende y, por lo tanto, el costo de selección adversa aumenta lentamente.

GRÁFICO A.6

\section{Comportamiento del costo de negociación de AUSTRAC1}

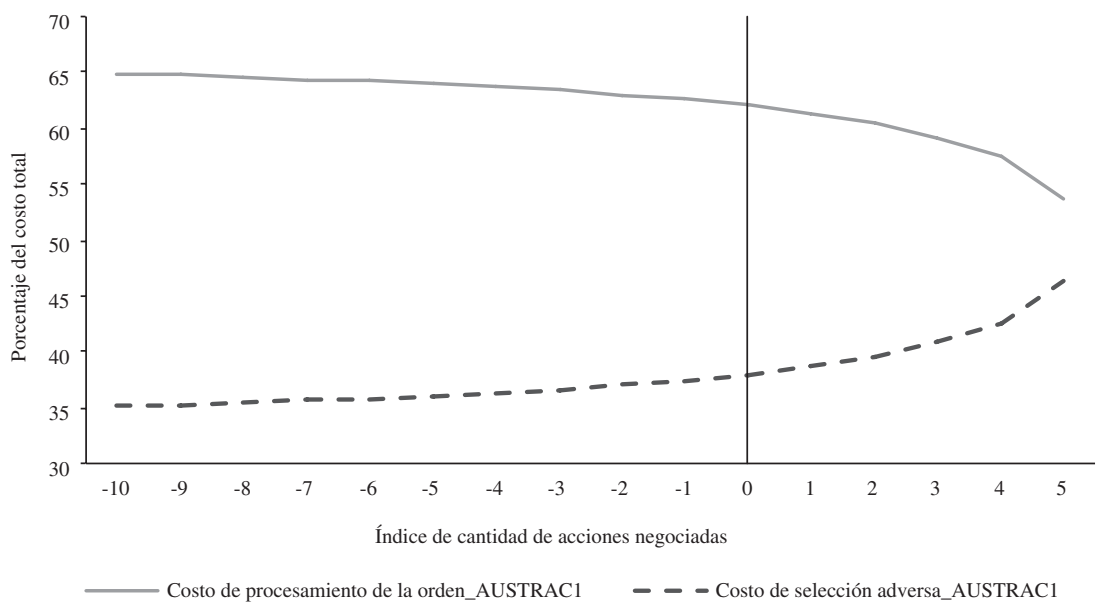

Fuente: elaboración propia.

En el gráfico A.7 se muestra el comportamiento de los componentes de los costos de la acción de EDELNOC1. Puede observarse que, conforme se incrementa el índice del número de acciones negociadas, el costo de procesamiento desciende rápidamente $\mathrm{y}$, por lo tanto, el costo de selección adversa aumenta.

GRÁFICO A.7

Comportamiento del costo de negociación de EDELNOC1

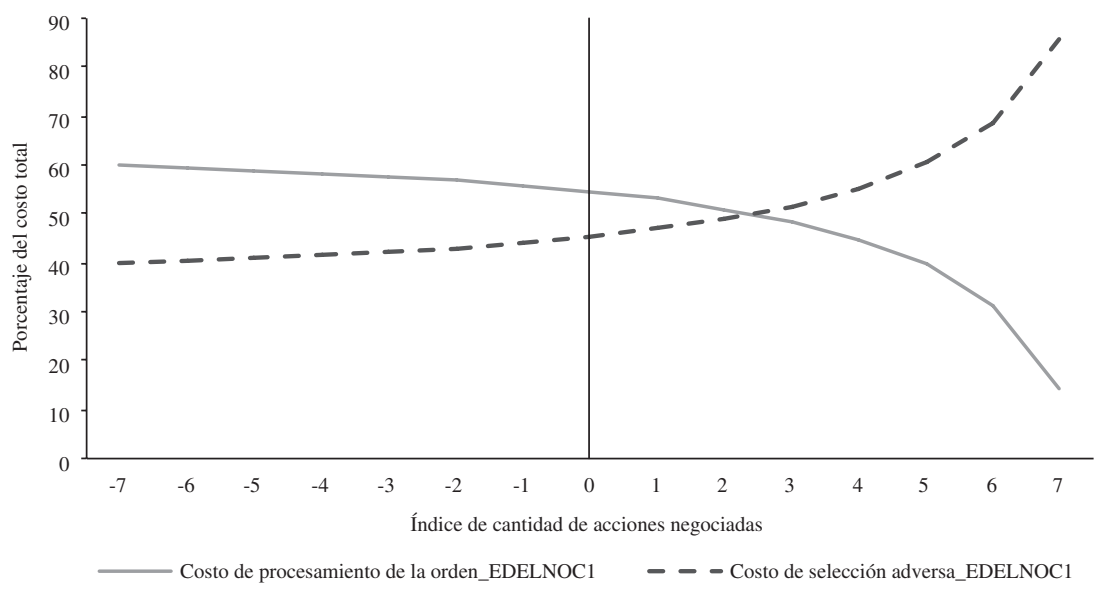

Fuente: elaboración propia. 
En el gráfico A.8 se muestra el comportamiento de los componentes de los costos de la acción de scotiac1. Puede observarse que, conforme se incrementa el índice del número de acciones negociadas, el costo de procesamiento desciende rápidamente $\mathrm{y}$, por lo tanto, el costo de selección adversa aumenta. Un aspecto curioso es que alcanza un nivel en el que se revierte la tendencia; por lo que sería interesante modelar estos componentes bajo una metodología no lineal, ya que se observa que posiblemente posea dos quiebres.

GRÁFICO A.8

Comportamiento del costo de negociación de scotiac1

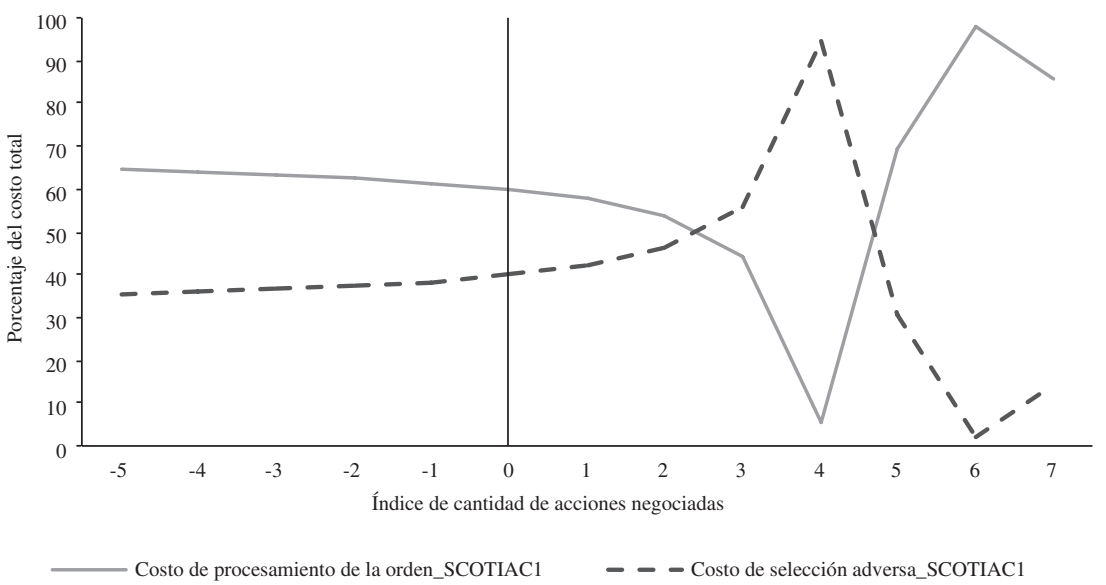

Fuente: elaboración propia. 
ANEXO B

GRÁFICO B.1

Funciones de impulso-respuesta del modelo econométrico VAR para RIO

Respuesta de D_PT_RIO a D_PT_RIO

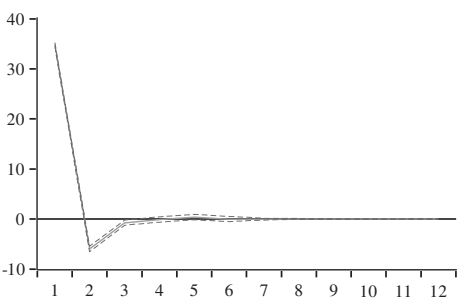

Respuesta de SIGNO a D_PT_RIO

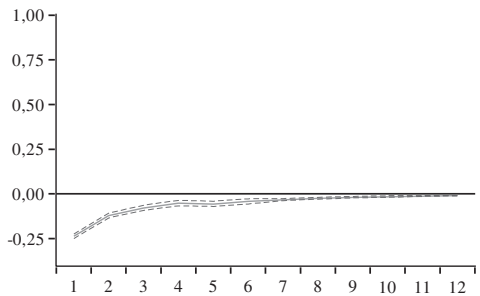

Respuesta de SIGNO_QT_INDEX a D_PT_RIO

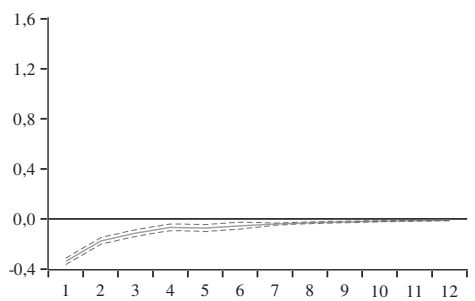

Respuesta de D_PT_RIO a SIGNO

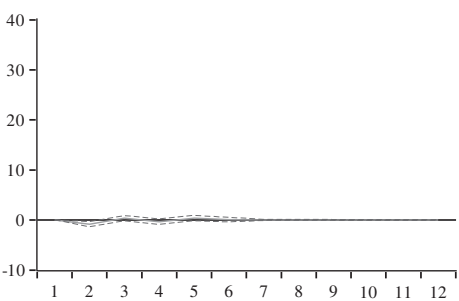

Respuesta de SIGNO a SIGNO

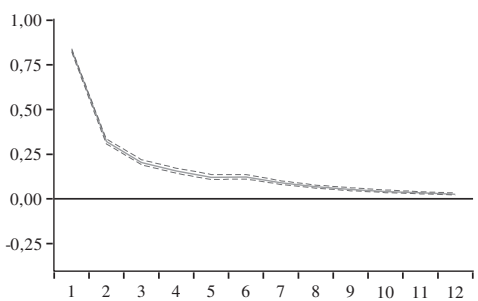

Respuesta de SIGNO_QT_INDEX a SIGNO

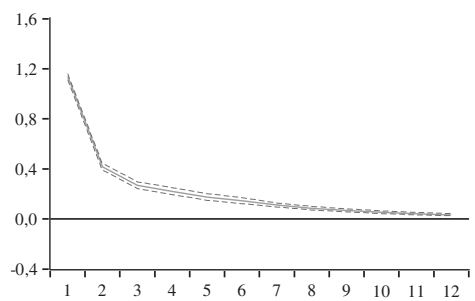

Respuesta de D_PT_RIO a SIGNO_QT_INDEX

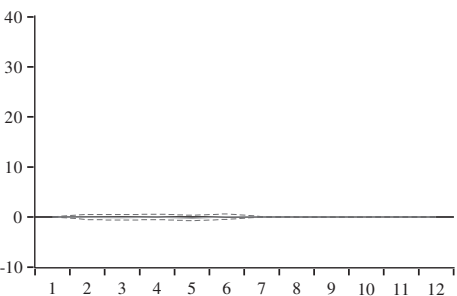

Respuesta de SIGNO a SIGNO_QT_INDEX

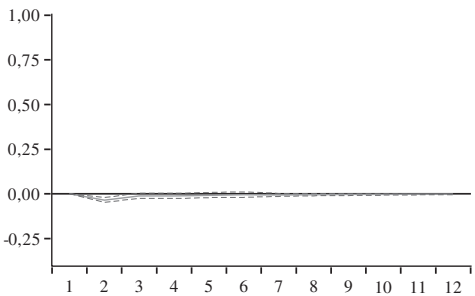

Respuesta de SIGNO_QT_INDEX a SIGNO_QT_INDEX

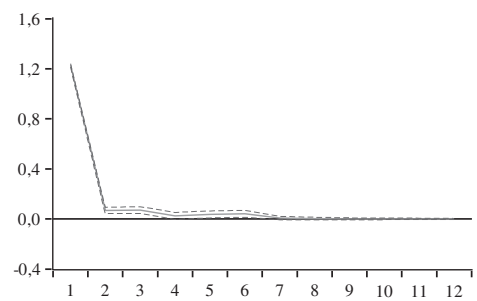

Fuente: elaboración propia. 
GRÁFICO B.2

Funciones de impulso-respuesta del modelo econométrico VAR para FERREYC1
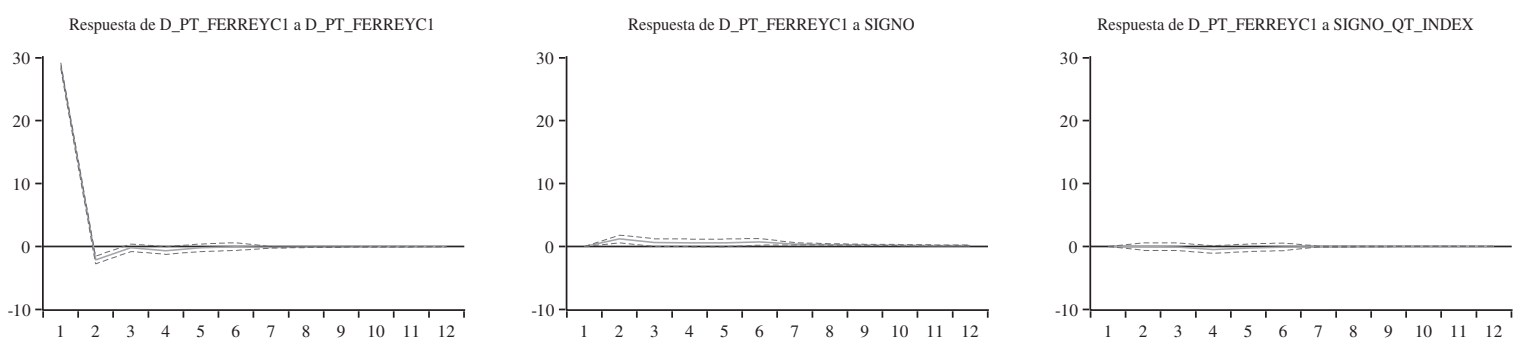

Respuesta de SIGNO a D_PT_FERREYC1
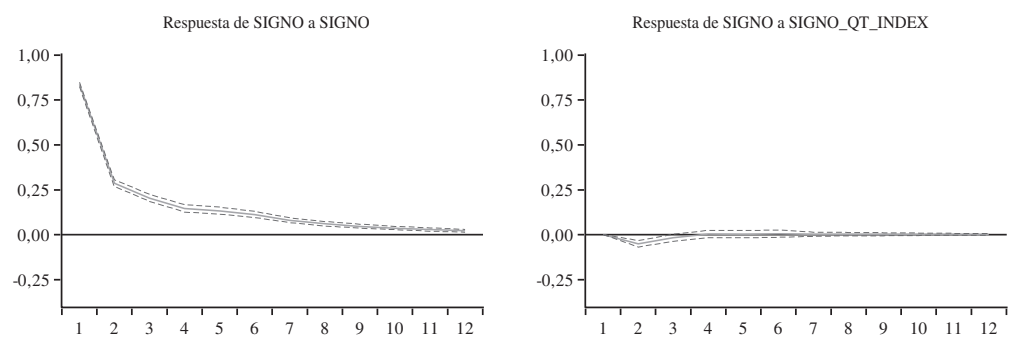

Respuesta de SIGNO_QT_INDEX a D_PT_FERREYCl

Respuesta de SIGNO_QT_INDEX a SIGNO
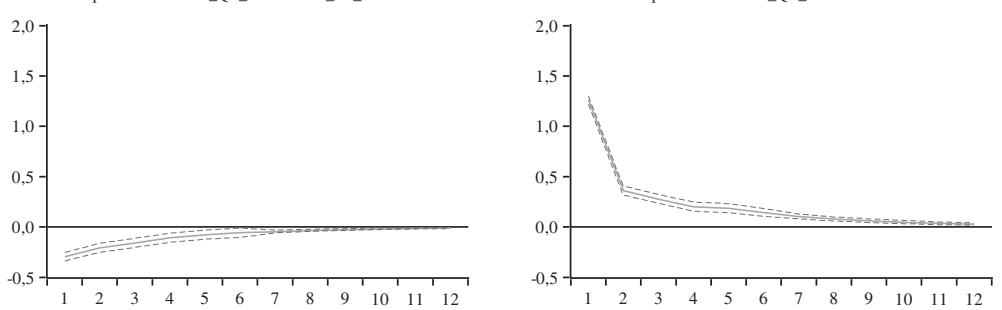

Respuesta de SIGNO_QT_INDEX a SIGNO_QT_INDEX

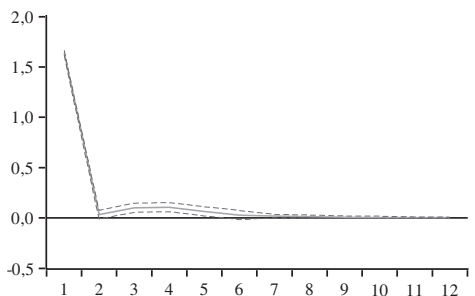

Fuente: elaboración propia. 
GRÁFICO B.3

Funciones de impulso-respuesta del modelo econométrico VAR para CVERDEC1

Respuesta de D_PT_CVERDEC1 a D_PT_CVERDEC1

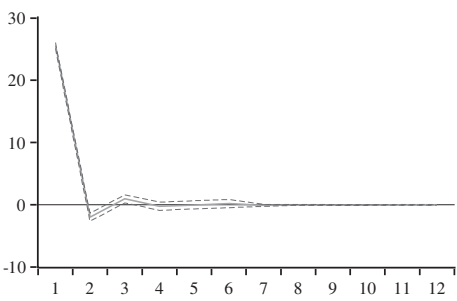

Respuesta de SIGNO a D PT CVERDEC1

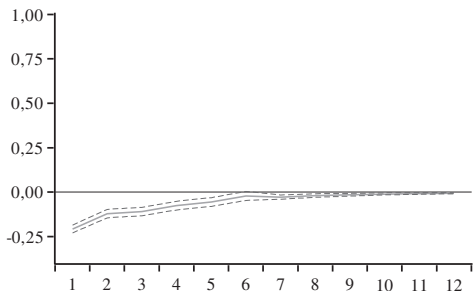

Respuesta de SIGNO_QT_INDEX a D_PT_CVERDEC1

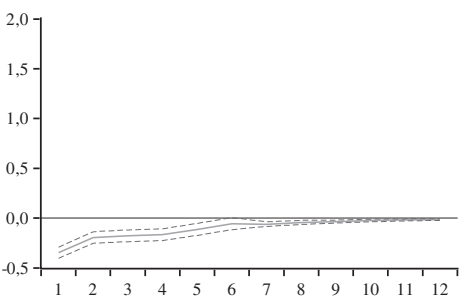

Fuente: elaboración propia.
Respuesta de D_PT_CVERDEC1 a SIGNO

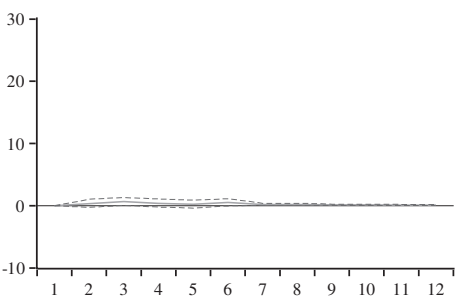

Respuesta de SIGNO a SIGNO

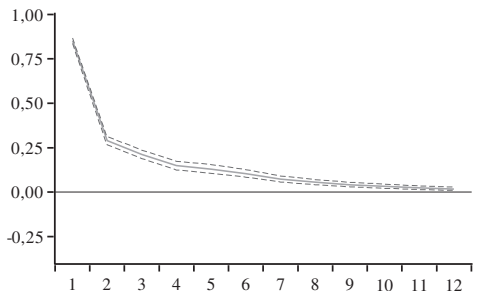

Respuesta de SIGNO_QT_INDEX a SIGNO

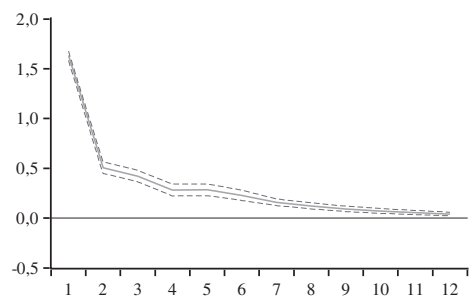

Respuesta de D_PT_CVERDEC1 a SIGNO_QT_INDEX

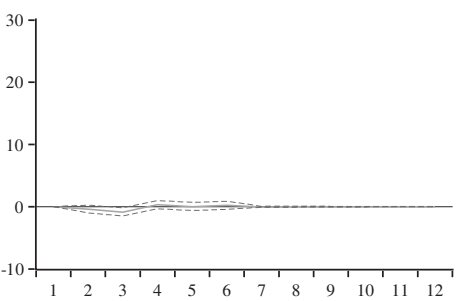

Respuesta de SIGNO a SIGNO_QT_INDEX

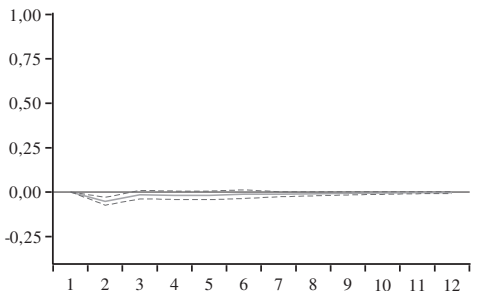

Respuesta de SIGNO_QT_INDEX a SIGNO_QT_INDEX

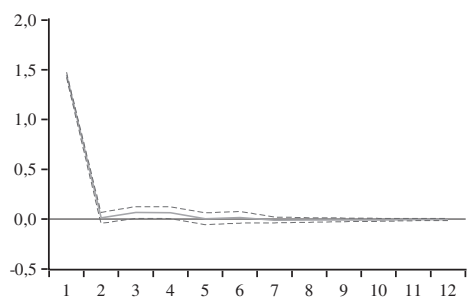


GRÁFICO B.4

\section{Funciones de impulso-respuesta del modelo econométrico VAR para BVN}

Respuesta de D_PT_BVN a D_PT_BVN

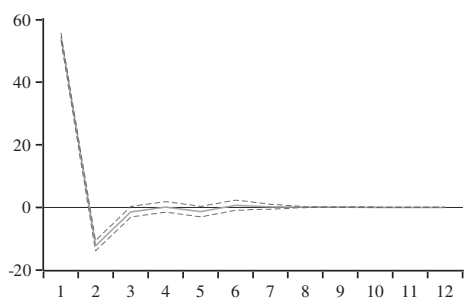

Respuesta de SIGNO a D_PT_BVN

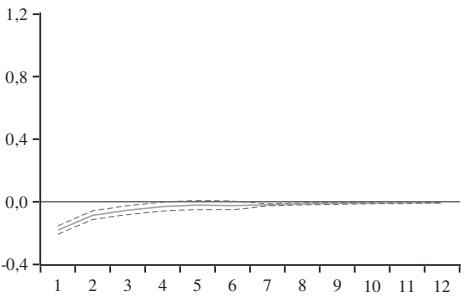

Respuesta de SIGNO_QT_INDEX a D_PT_BVN

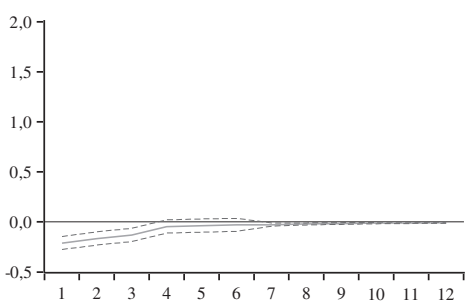

Respuesta de D_PT_BVN a SIGNO

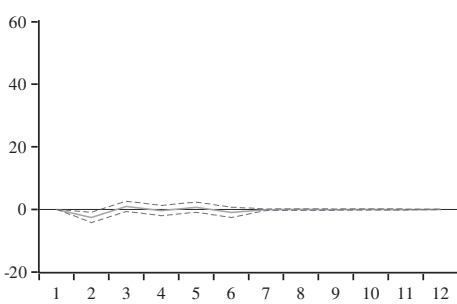

Respuesta de SIGNO a SIGNO

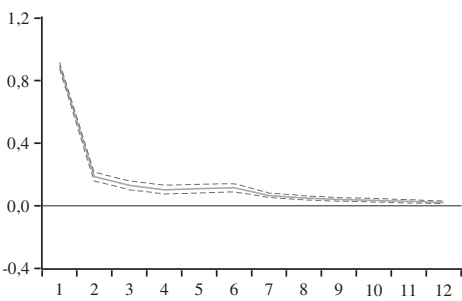

Respuesta de SIGNO_QT_INDEX a SIGNO

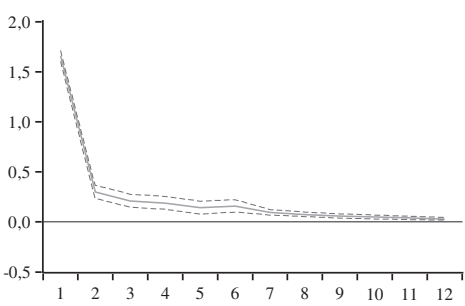

Respuesta de D_PT_BVN a SIGNO_QT_INDEX

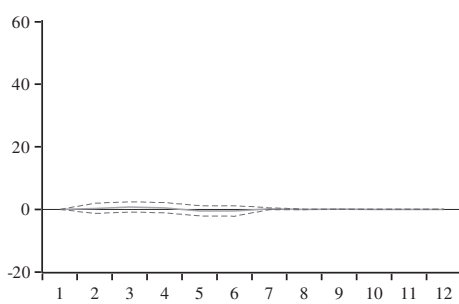

Respuesta de SIGNO a SIGNO_QT_INDEX

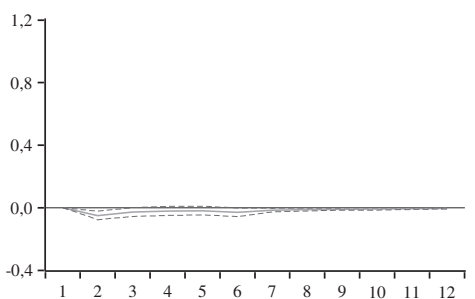

Respuesta de SIGNO_QT_INDEX a SIGNO_QT_INDEX

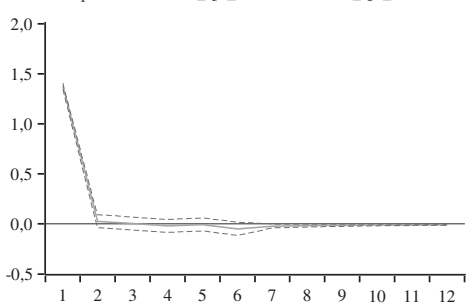

Fuente: elaboración propia. 
GRÁFICO B.5

Funciones de impulso-respuesta del modelo econométrico VAR para BROCALC1
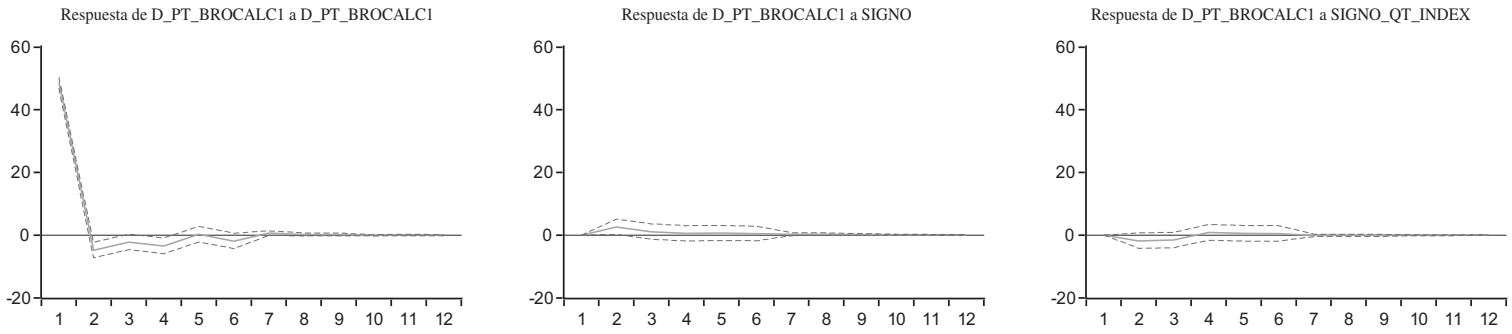

Respuesta de SIGNO a D_PT_BROCALC1
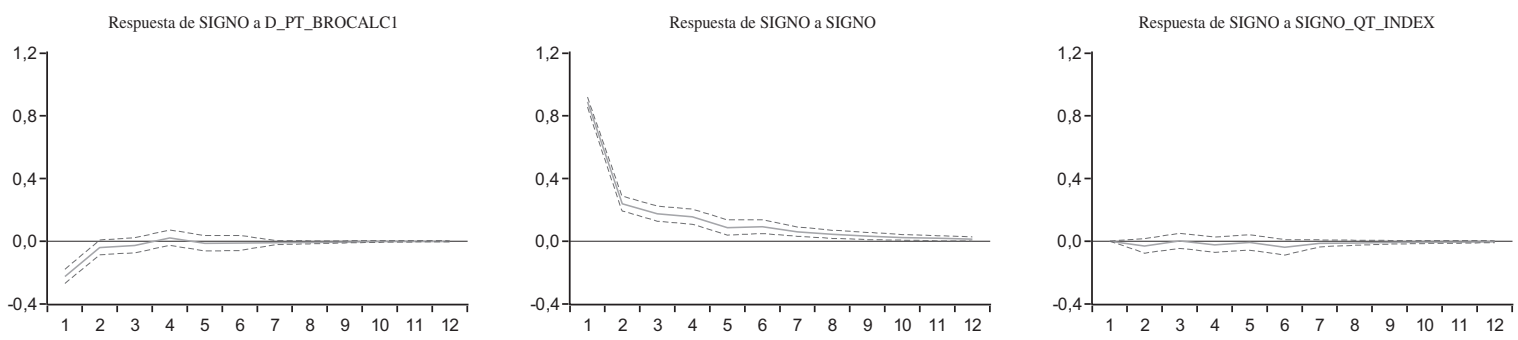

Respuesta de SIGNO_QT_INDEX a SIGNO
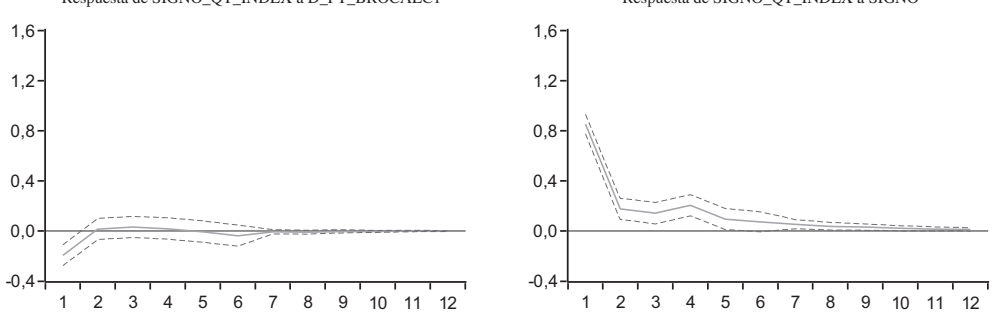

Respuesta de SIGNO_QT_INDEX a SIGNO_QT_INDEX

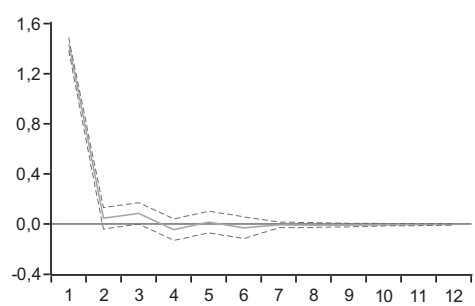

Fuente: elaboración propia. 
GRÁFICO B.6

Funciones de impulso-respuesta del modelo econométrico VAR para AUSTRAC1
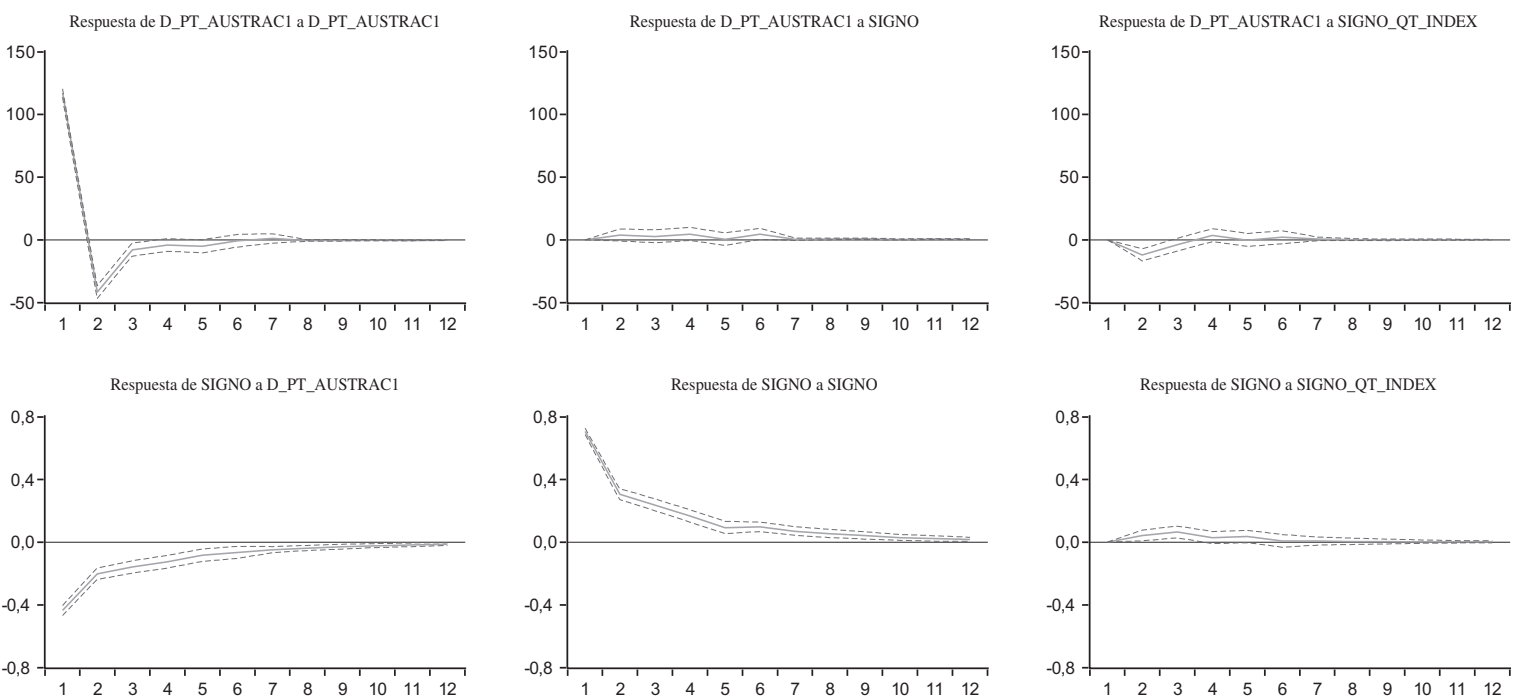

Respuesta de SIGNO_QT_INDEX a D_PT_AUSTRAC1

Respuesta de SIGNO_QT_INDEX a SIGNO
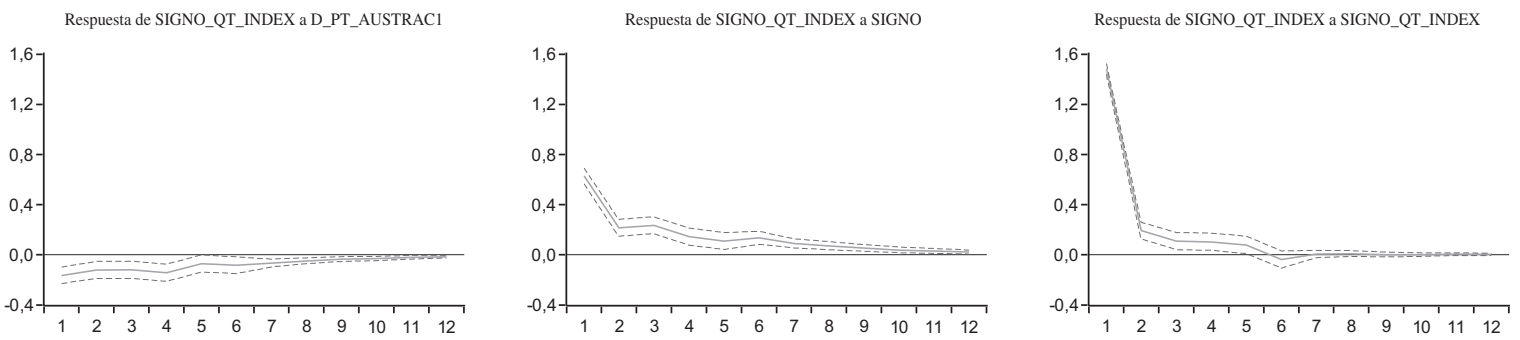

Fuente: elaboración propia. 
GRÁFICO B.7

Funciones de impulso-respuesta del modelo econométrico VAR para POMALCC1

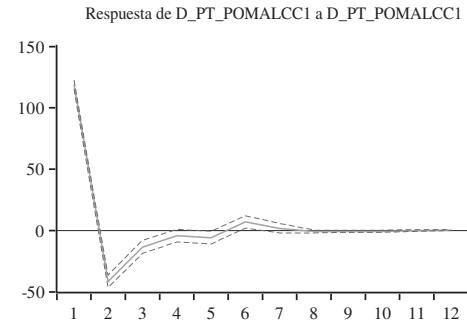

Respuesta de SIGNO a D_PT_POMALCC1

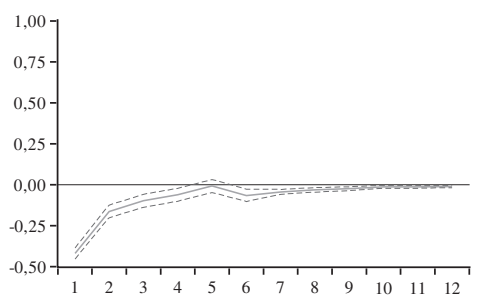

Respuesta de SIGNO_QT_INDEX a D_PT_POMALCC1

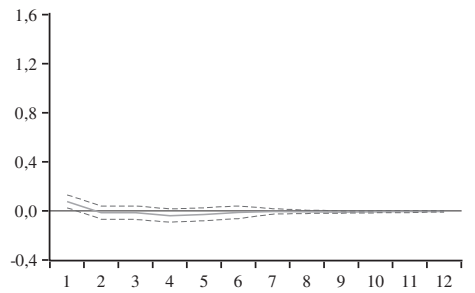

Respuesta de D_PT_POMALCC1 a SIGNO

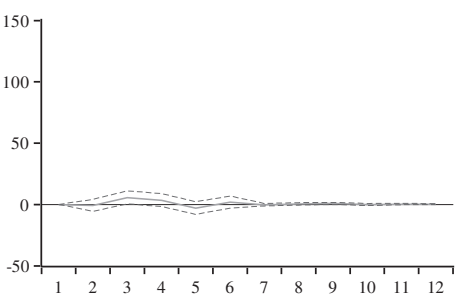

Respuesta de SIGNO a SIGNO

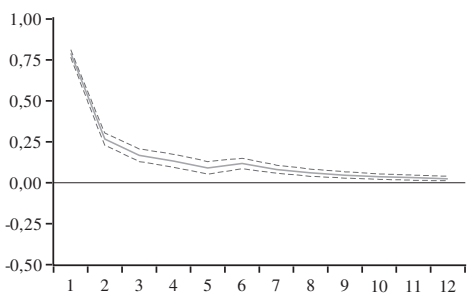

Respuesta de SIGNO_QT_INDEX a SIGNO

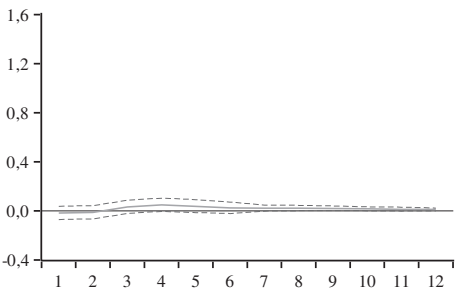

Respuesta de D_PT_POMALCC1 a SIGNO_QT_INDEX

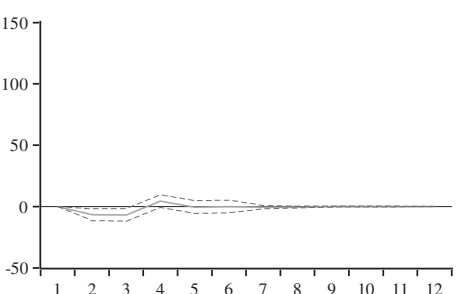

Respuesta de SIGNO a SIGNO QT INDEX

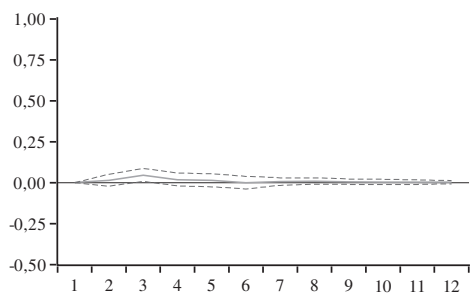

Respuesta de SIGNO QTT_INDEX a SIGNO_QT_INDEX

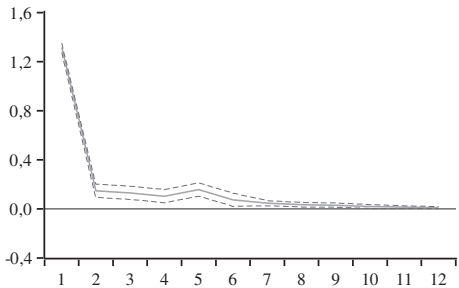

Fuente: elaboración propia. 
GRÁFICO B.8

Funciones de impulso-respuesta del modelo econométrico VAR para EDELNOC1
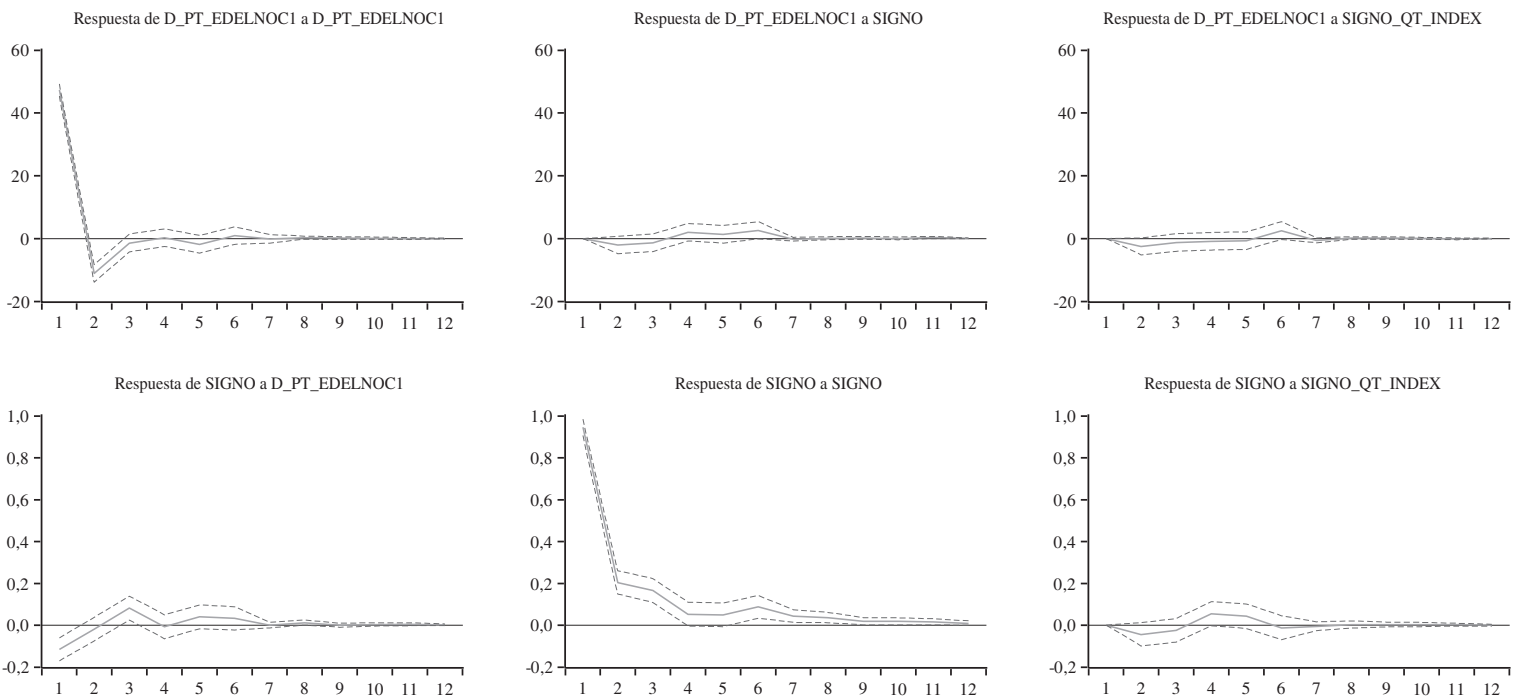

Respuesta de SIGNO a SIGNO_QT_INDEX

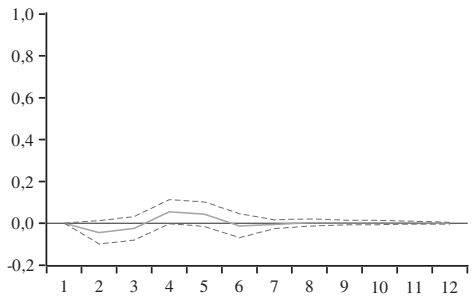

Respuesta de SIGNO_QT_INDEX a D_PT_EDELNOC1

Respuesta de SIGNO_QT_INDEX a SIGNO

Respuesta de SIGNO_QT_INDEX a SIGNO_QT_INDEX
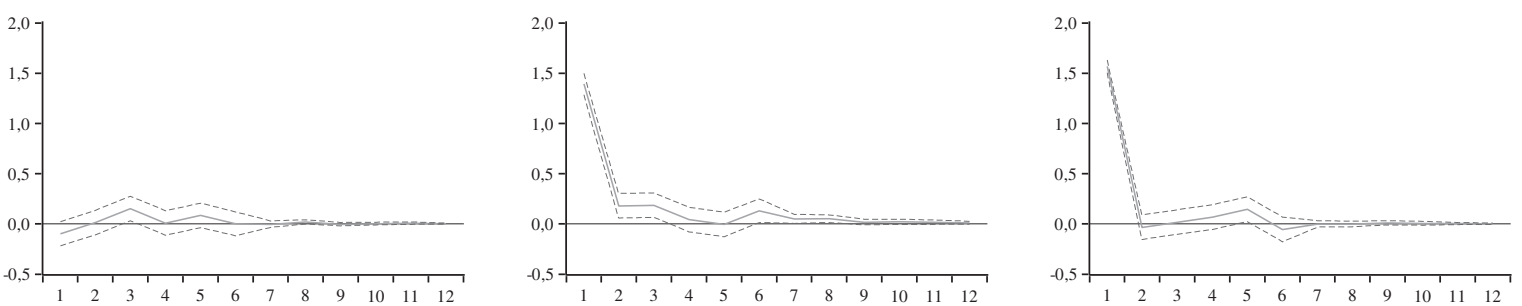

Fuente: elaboración propia. 
GRÁFICO B.9

Funciones de mpulso-respuesta del modelo econométrico VAR para SCOTIAC1

Respuesta de D_PT_SCOTIACl a D_PT_SCOTIAC1

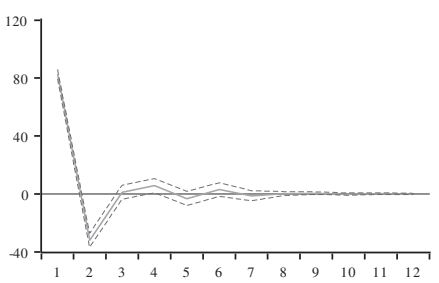

Respuesta de SIGNO a D_PT_SCOTIACI

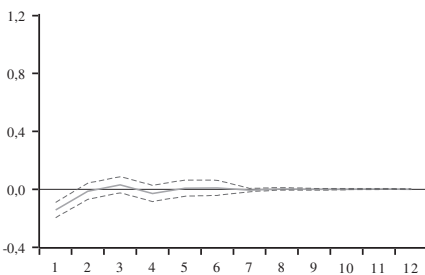

Respuesta de SIGNO_QT_INDEX a D P PT_SCOTIACI

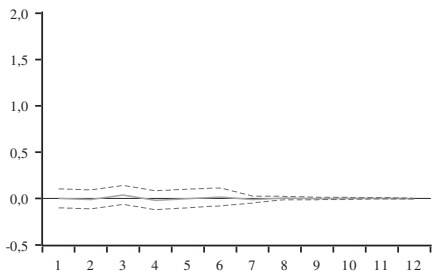

Respuesta de D_PT_SCOTIAC1 a SIGNO

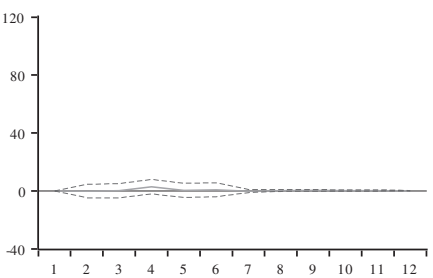

Respuesta de SIGNO a SIGNO

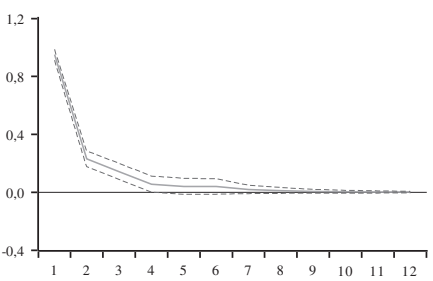

Respuesta de SIGNO_QT_INDEX a SIGNO

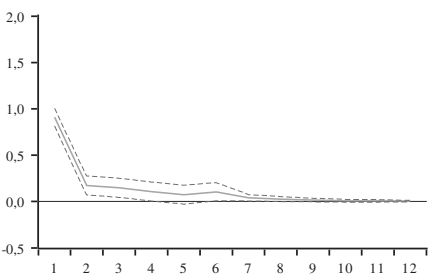

Respuesta de D_PT_SCOTIAC1 a SIGNO_QT_INDEX

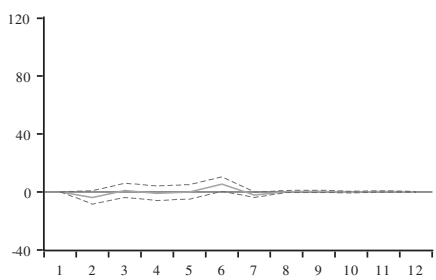

Respuesta de SIGNO a SIGNO_QT_INDEX

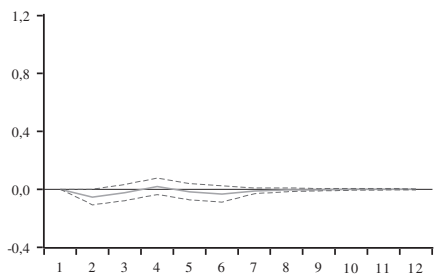

Respuesta de SIGNO_QT_INDEX a SIGNO_QT_INDEX

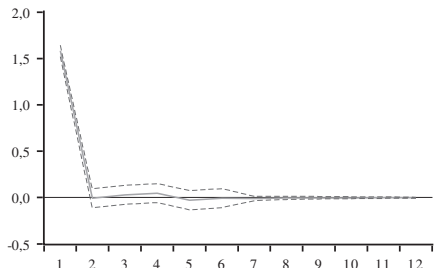

Fuente: elaboración propia. 


\section{Bibliografía}

Black, F. (1971), "Toward a fully automated exchange, Part 1", Financial Analysts Journal, vol. 27, $\mathrm{N}^{\circ}$ 4, CFA Institute.

Bolsa de Valores de Lima (2012), "Cartera del Índice General BVL" [en línea] http://www.bvl.com.pe/estadist/IndGral.htm.

Cochrane, J. (1988), "How big is the random walk in GNP?", Journal of Political Economy, vol. 96, $\mathrm{N}^{\circ}$ 5, Chicago, University of Chicago Press.

Demsetz, H. (1968), "The cost of transacting", Quarterly Journal of Economics, vol. 82, $\mathrm{N}^{\circ}$ 1, Oxford, Oxford University Press.

Easley, D. y otros (2008), "Time-varying arrival rates of informed and uninformed trades", Journal of Financial Econometrics, vol. $6, \mathrm{~N}^{\circ} 2$, Oxford, Oxford University Press.

Foucault, T., O. Kadan y E. Kandel (2001), "Limit order book as a market for liquidity", The Review of Financial Studies, vol. 18, $\mathrm{N}^{\circ}$ 4, Oxford, Oxford University Press.

Garman, M. (1976), "Market microstructure", Journal of Financial Economics, vol. 3, $\mathrm{N}^{\circ} 3$, Amsterdam, Elsevier.

Glosten, L. (1994), "Is the electronic open limit order book inevitable?", Journal of Finance, vol. 49, $\mathrm{N}^{\circ} 4$, Wiley.

Glosten, L. y L. Harris (1988), "Estimating the components of the bid/ask spread", Journal of Financial Economics, vol. 21, Nㅜㄹ, Amsterdam, Elsevier.

Glosten, L. y P. Milgrom (1985), "Bid, ask and transaction prices in a specialist market with heterogeneously informed traders", Journal of Financial Economics, vol. 14, № 1, Amsterdam, Elsevier.

Harris, L. (1986), "A transaction data study of weekly and intradaily patterns in stock returns", Journal of Financial Economics, vol. 16, $\mathrm{N}^{\circ} 1$, Amsterdam, Elsevier.

Hasbrouck, J. (1993), "Assessing the quality of a security market: a new approach to transaction cost measurement", Review of Financial Studies, vol. 6, $\mathrm{N}^{\circ}$ 1, Oxford, Oxford University Press. (1991a), "Measuring the information content of stock trades", Journal of Finance, vol. 46, $\mathrm{N}^{\circ} 1$, Wiley.

(1991b), "The summary informativeness of stock trades: an econometric analysis", Review of Financial Studies, vol. 4, $\mathrm{N}^{\circ} 3$, Oxford, Oxford University Press.

(1988), "Trades, quotes, inventories and information", Journal of Financial Economics, vol. 22, № 2, Amsterdam, Elsevier.
Heckman, J. (1978), "Dummy endogenous variables in a simultaneous equation system", Econometrica, vol. 46, ํㅜ 4, Econometric Society.

Jong, F., T. Nijman y A. Röell (1996), "Price effects of trading and components of the bid-ask spread on the Paris Bourse", Journal of Empirical Finance, vol. 3, $\mathrm{N}^{\circ}$ 2, Amsterdam, Elsevier.

Kyle, A.S. (1985), "Continuous auctions and insider trading", Econometrica, vol. 53, № 6 , The Econometric Society.

Loaiza, C. (2013), "Relación entre liquidez y la microestructura del mercado de renta variable al contado, en la Bolsa de Valores de Lima", tesis [en línea] http://tesis.pucp.edu.pe/repositorio/ handle/123456789/6.

Madhavan, A., M. Richardson y M. Roomans (1994), "Why do security prices change? A transaction level analysis of NYSE stocks", The Review of Financial Studies, vol. 10, ํㅜ 4, Oxford, Oxford University Press.

Markowitz, H. (1952), "Portfolio selection", Journal of Finance, vol. $7, \mathrm{~N}^{\circ} 1$, Wiley.

Newey, W. y L. West (1987), "A simple, positive semi-definite, heteroskedasticity and autocorrelation consistent covariance matrix", Econometrica, vol. 55, №3, Econometric Society.

O'Hara, M. (1995), Market Microstructure Theory, Cambridge, Massachusetts, Blackwell Business.

Rosu, I. (2009), "A dynamic model of the limit order book", Review of Financial Studies, vol. 22, N 11, Oxford, Oxford University Press.

Sharpe, W. (1964), "Capital asset prices: a theory of market equilibrium under conditions of risk", Journal of Finance, vol. $19, \mathrm{~N}^{\circ} 3$, Wiley.

Sims, C. (1980), "Macroeconomics and reality", Econometrica, vol. 48, $\mathrm{N}^{\circ} 1$, Econometric Society.

Stoll, H.R. (2000), "Friction", Journal of Finance, vol. 55, $\mathrm{N}^{\circ} 4$, Wiley.

(1989), "Inferring the components of the bid-ask spread: theory and empirical tests", Journal of Finance, vol. 44, $\mathrm{N}^{\circ} 1$, Wiley.

(1978), "The supply of dealer services in securities markets", Journal of Finance, vol. 33, № 4, Wiley. 